Supporting Information

\title{
Rh-Catalyzed Deformylative Coupling of Salicylaldehydes with Acrylates and Acrylamides
}

\author{
Maddali L. N. Rao* and Boddu S. Ramakrishna \\ Department of Chemistry, Indian Institute of Technology Kanpur, Kanpur-208016, India \\ Email: maddali@iitk.ac.in \\ Fax: +915122597532
}

Table of Contents

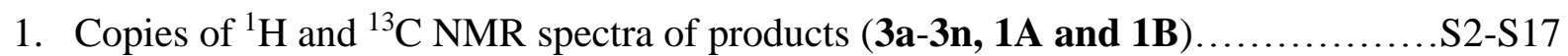

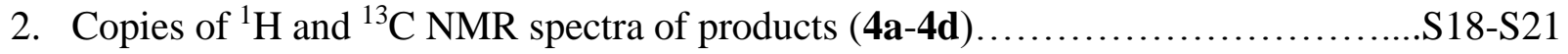

3. Copies of HRMS spectra of products $(\mathbf{3 a - 3 n , ~ 1 A , ~ 1 B ~ a n d ~ 4 a - 4 d ) \ldots . . . . . . . . . . . . . S 2 2 - S 4 1 ~}$ 


\section{Copies of ${ }^{1} \mathrm{H}$ and ${ }^{13} \mathrm{C}$ NMR spectra of products (3a-3n, $1 \mathrm{~A}$ and $\left.1 \mathrm{~B}\right)$}
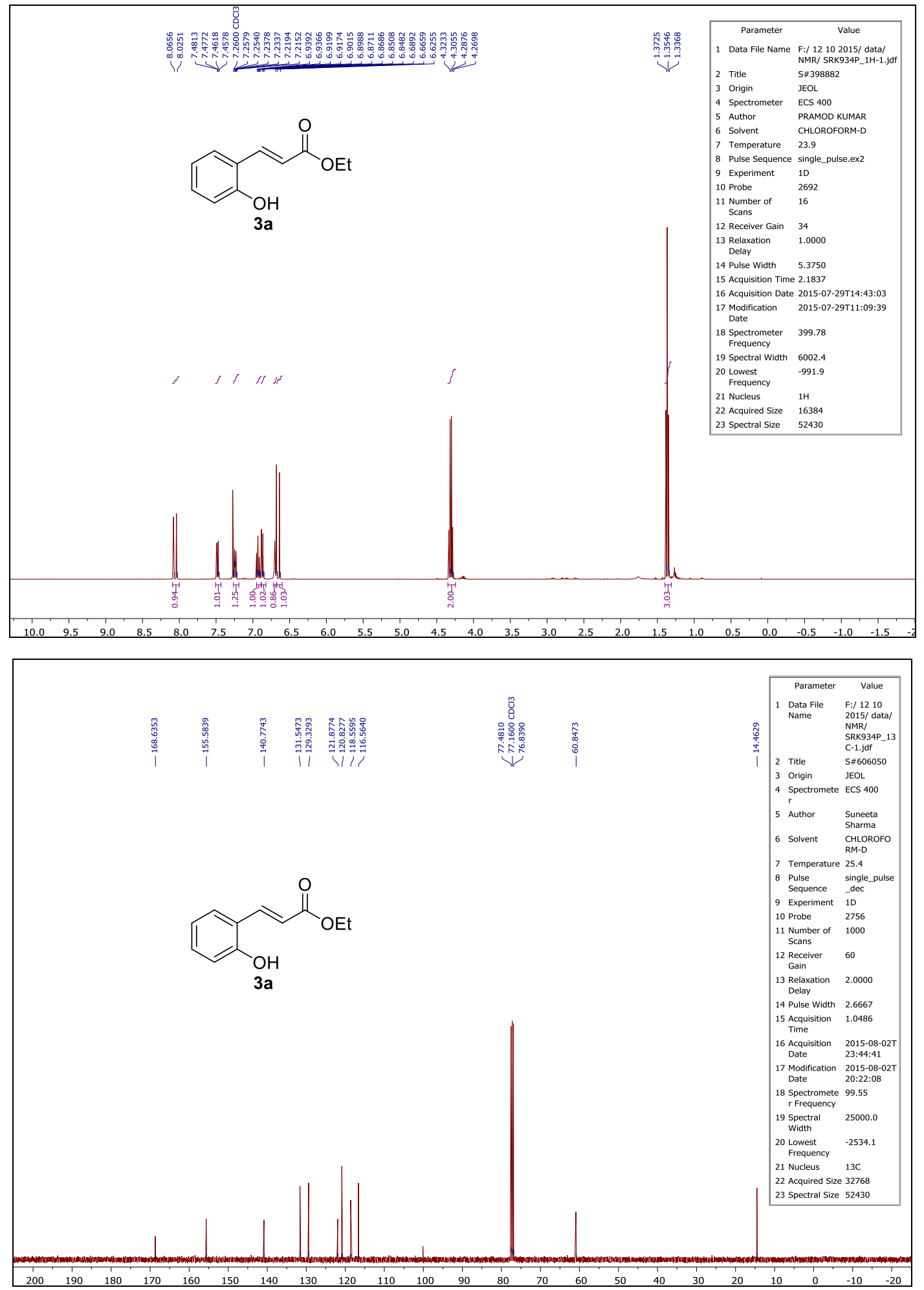

${ }^{1} \mathrm{H}$ and ${ }^{13} \mathrm{C}$ NMR spectra of (E)-ethyl 3-(2-hydroxyphenyl)acrylate (3a) 

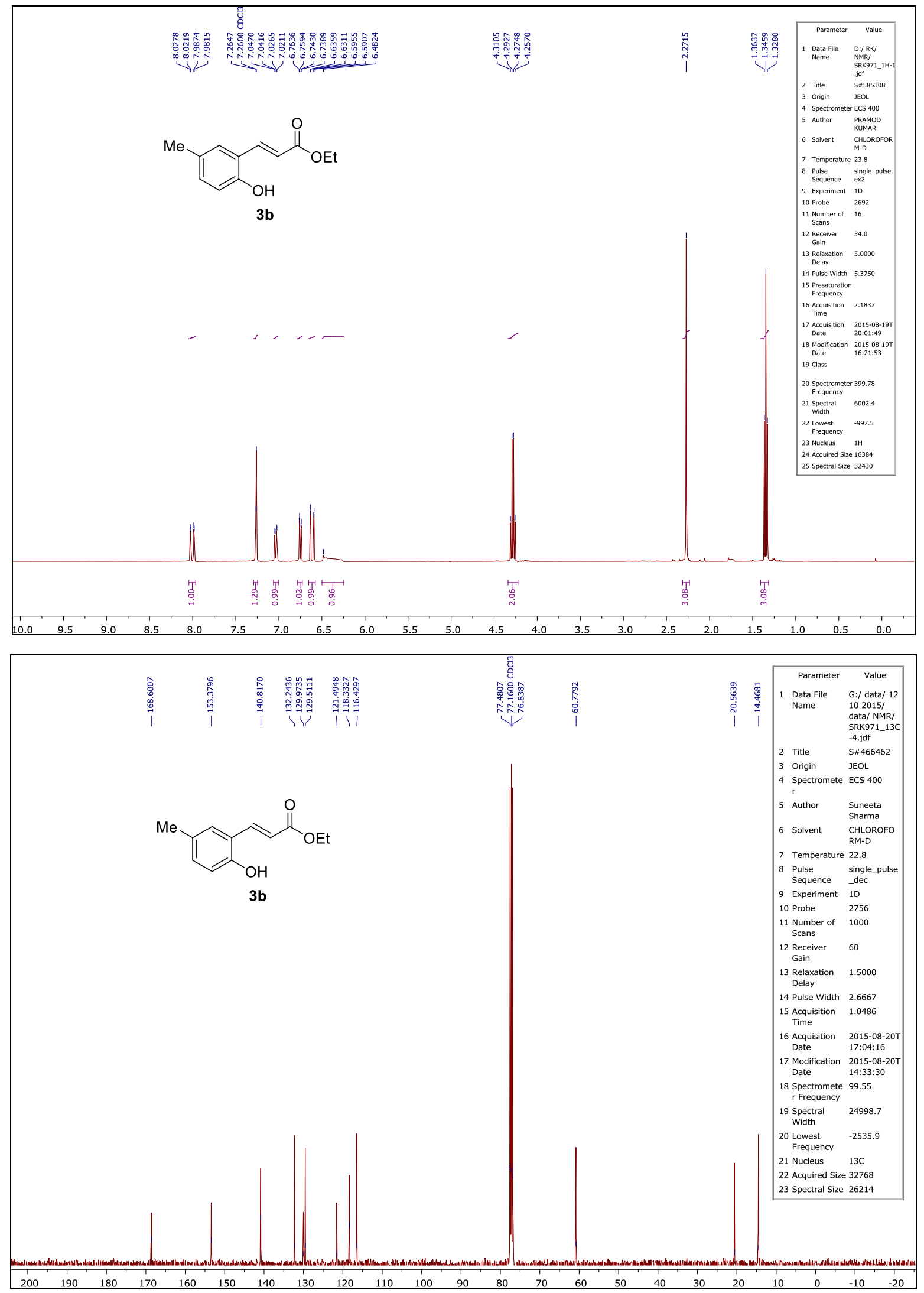

${ }^{1} \mathrm{H}$ and ${ }^{13} \mathrm{C}$ NMR spectra of (E)-ethyl 3-(2-hydroxy-5-methylphenyl)acrylate (3b) 

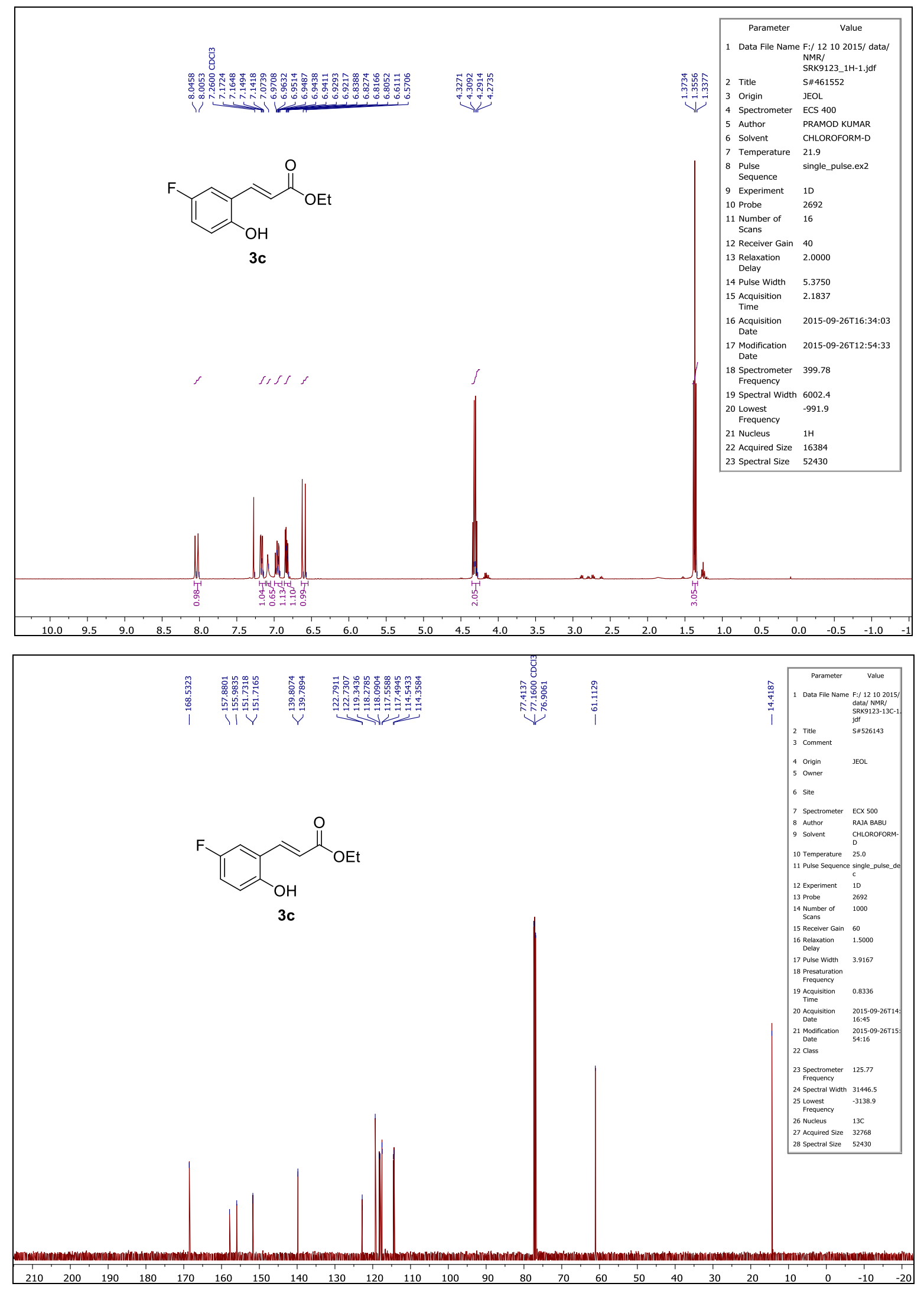

${ }^{1} \mathrm{H}$ and ${ }^{13} \mathrm{C}$ NMR spectra of (E)-ethyl 3-(5-fluoro-2-hydroxyphenyl)acrylate (3c) 

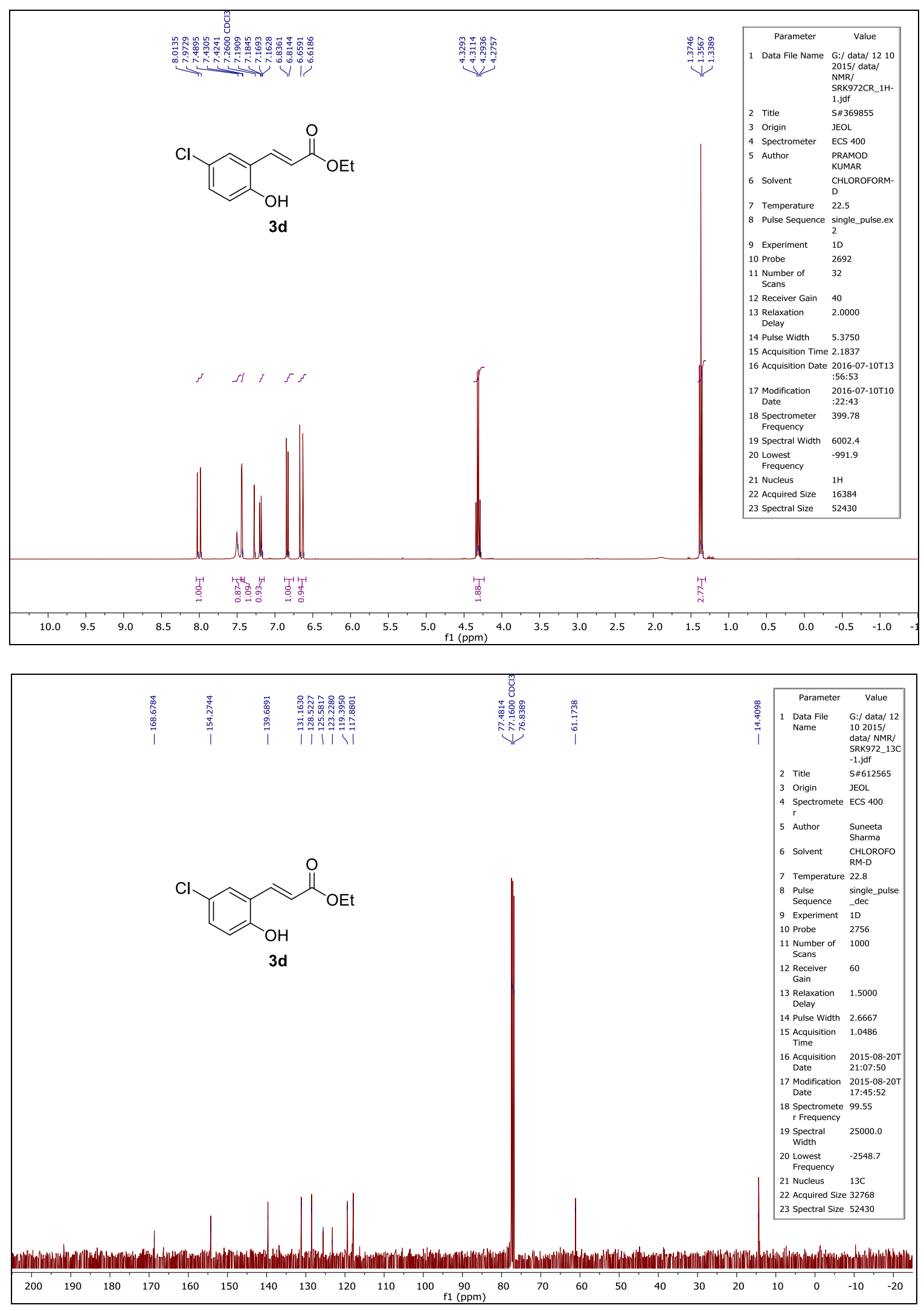

${ }^{1} \mathrm{H}$ and ${ }^{13} \mathrm{C}$ NMR spectrum of (E)-ethyl 3-(5-chloro-2-hydroxyphenyl)acrylate (3d) 

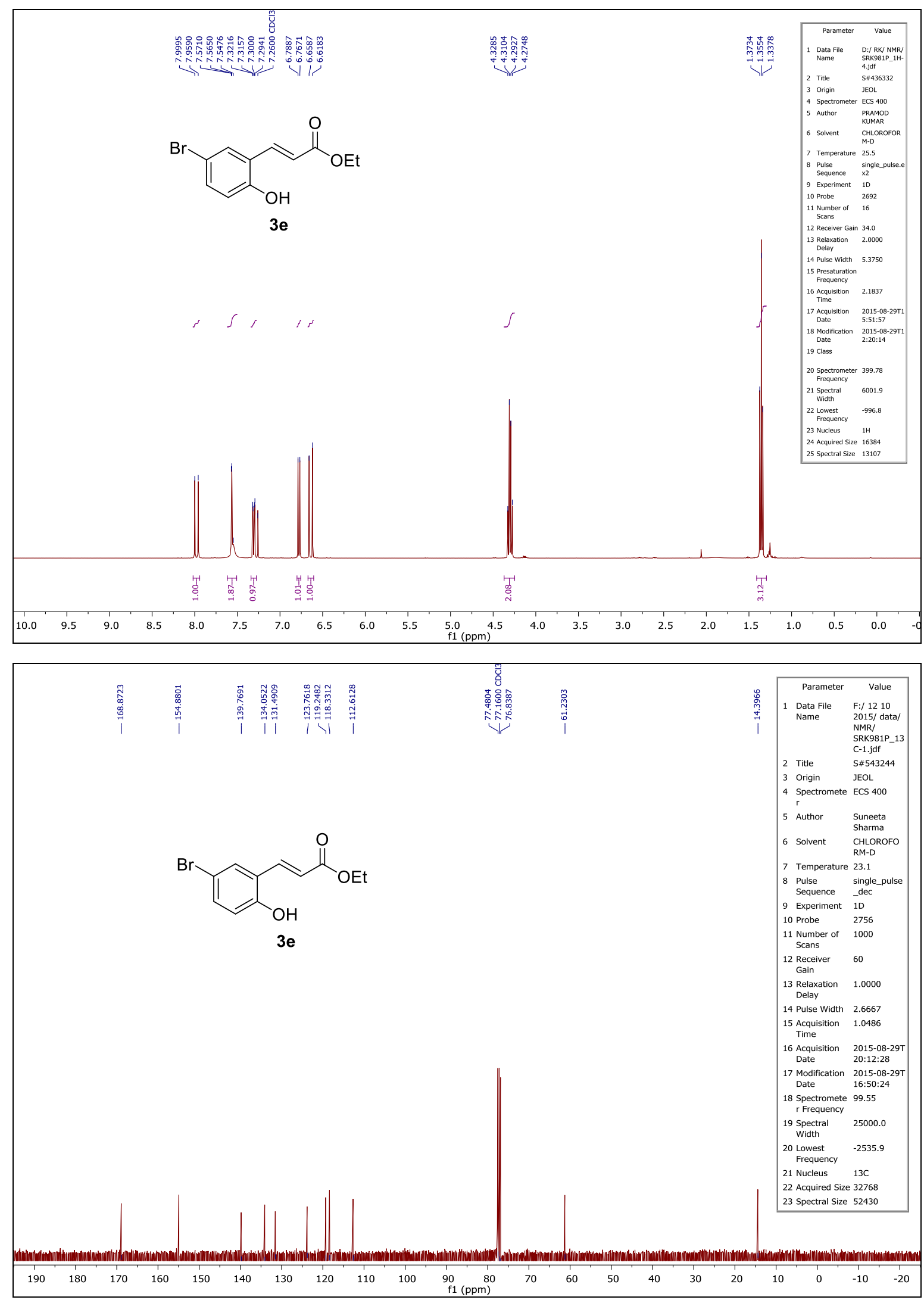

${ }^{1} \mathrm{H}$ and ${ }^{13} \mathrm{C}$ NMR spectrum of (E)-ethyl 3-(5-bromo-2-hydroxyphenyl)acrylate (3e) 


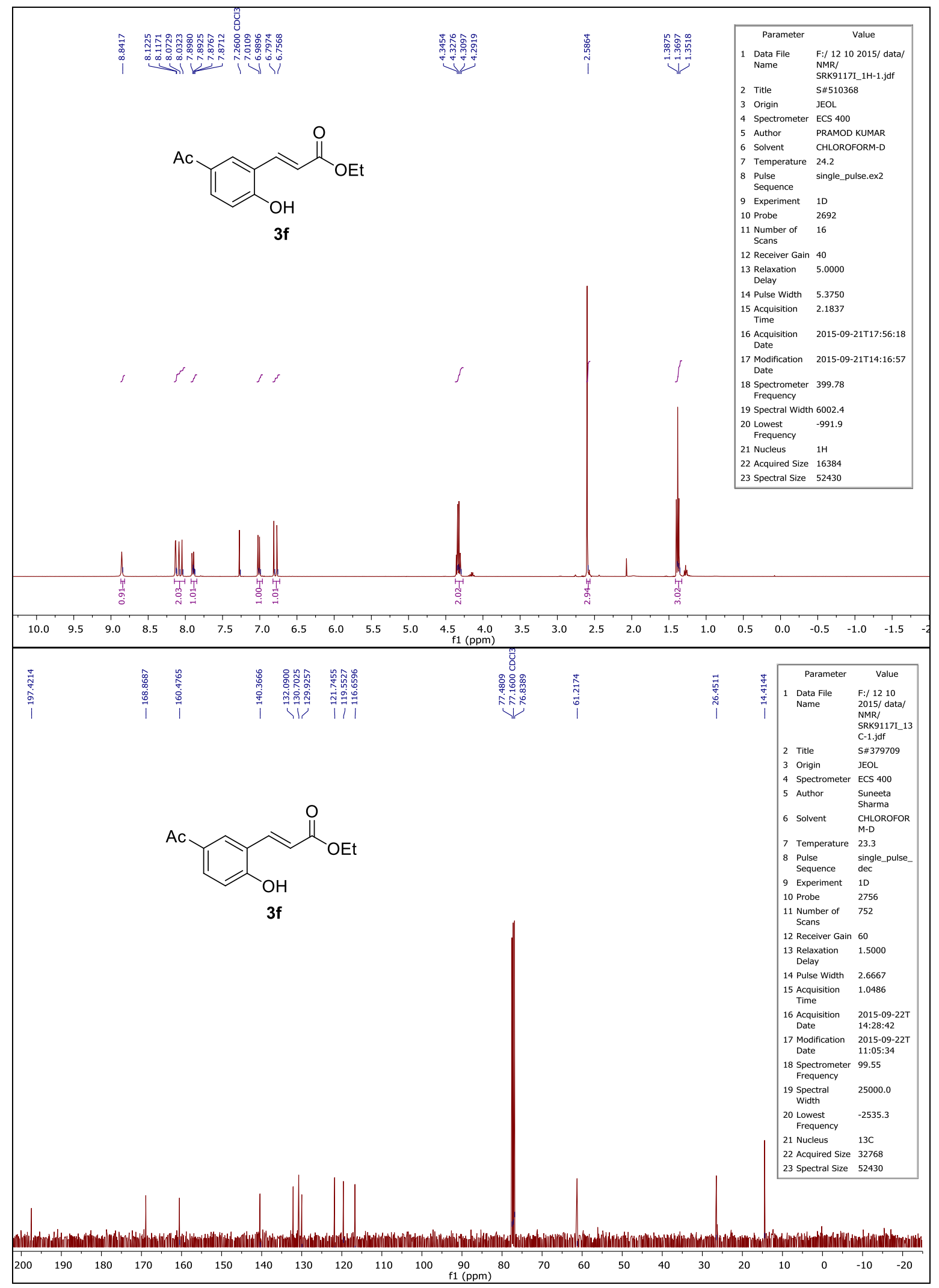

${ }^{1} \mathrm{H}$ and ${ }^{13} \mathrm{C}$ NMR spectrum of (E)-ethyl 3-(5-acetyl-2-hydroxyphenyl)acrylate (3f) 

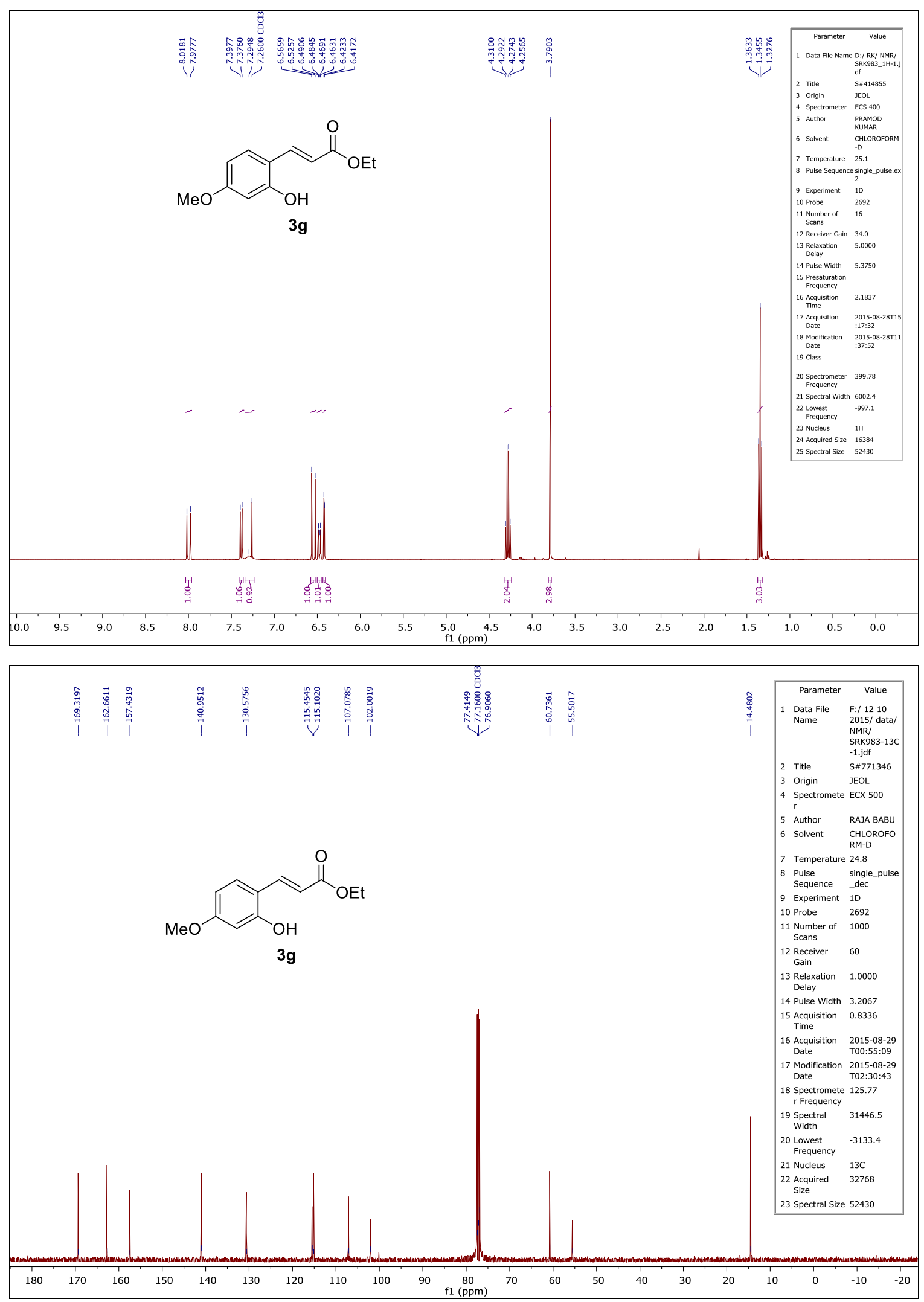

${ }^{1} \mathrm{H}$ and ${ }^{13} \mathrm{C}$ NMR spectrum of (E)-ethyl 3-(2-hydroxy-4-methoxyphenyl)acrylate (3g) 

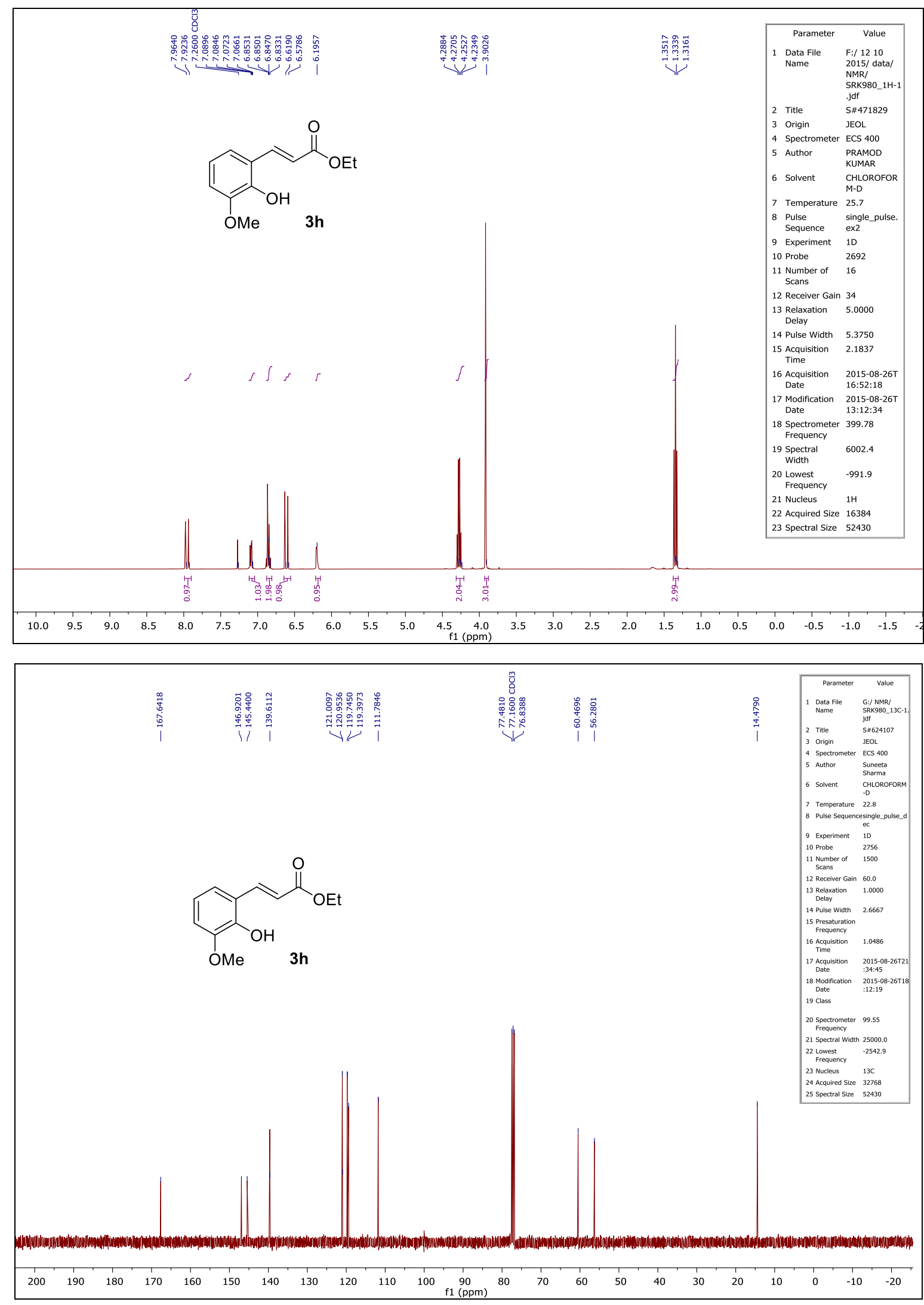

${ }^{1} \mathrm{H}$ and ${ }^{13} \mathrm{C}$ NMR spectrum of (E)-ethyl 3-(2-hydroxy-3-methoxyphenyl)acrylate (3h) 

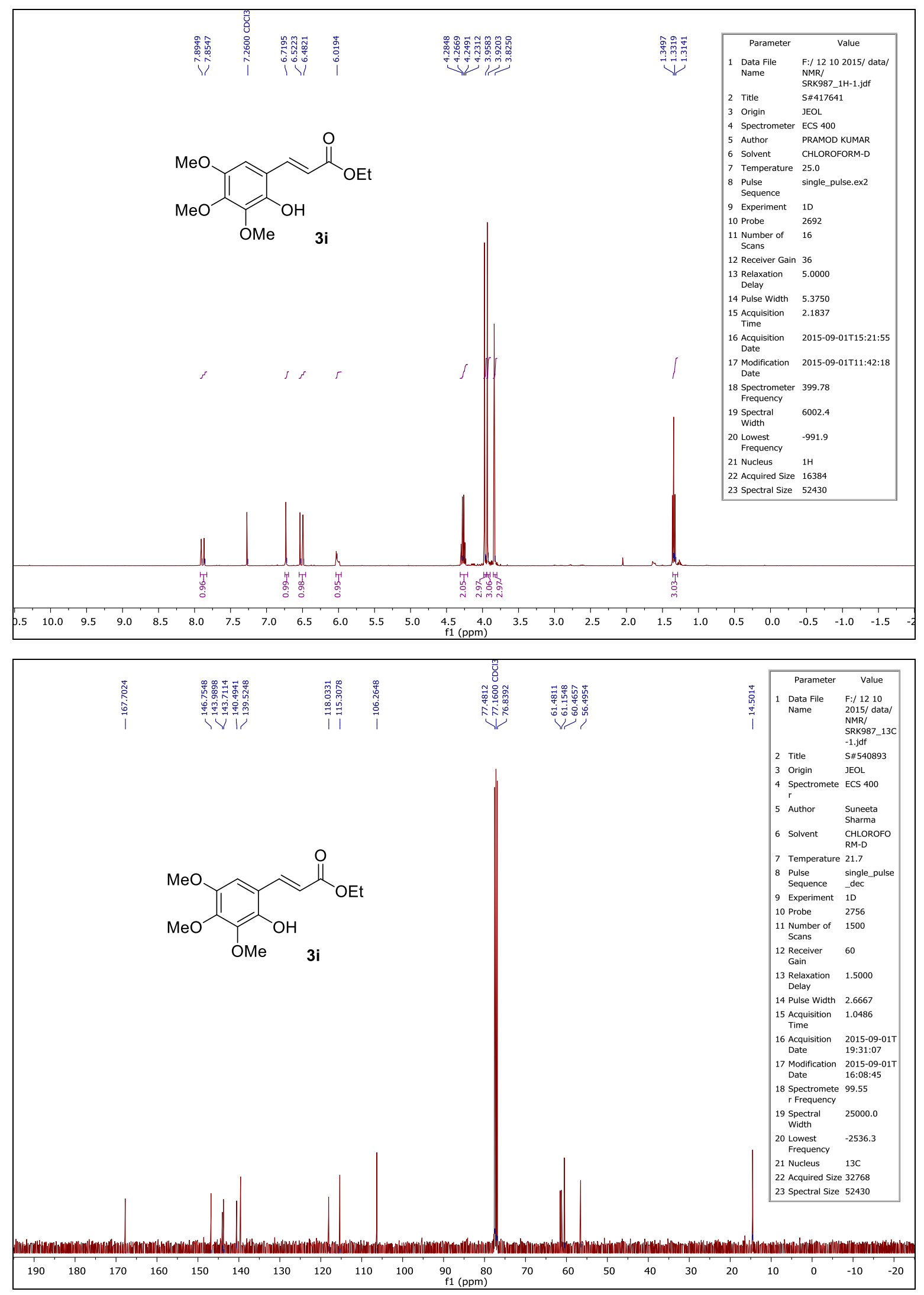

${ }^{1} \mathrm{H}$ and ${ }^{13} \mathrm{C}$ NMR spectrum of (E)-ethyl 3-(2-hydroxy-3,4,5-trimethoxyphenyl)acrylate (3i) 


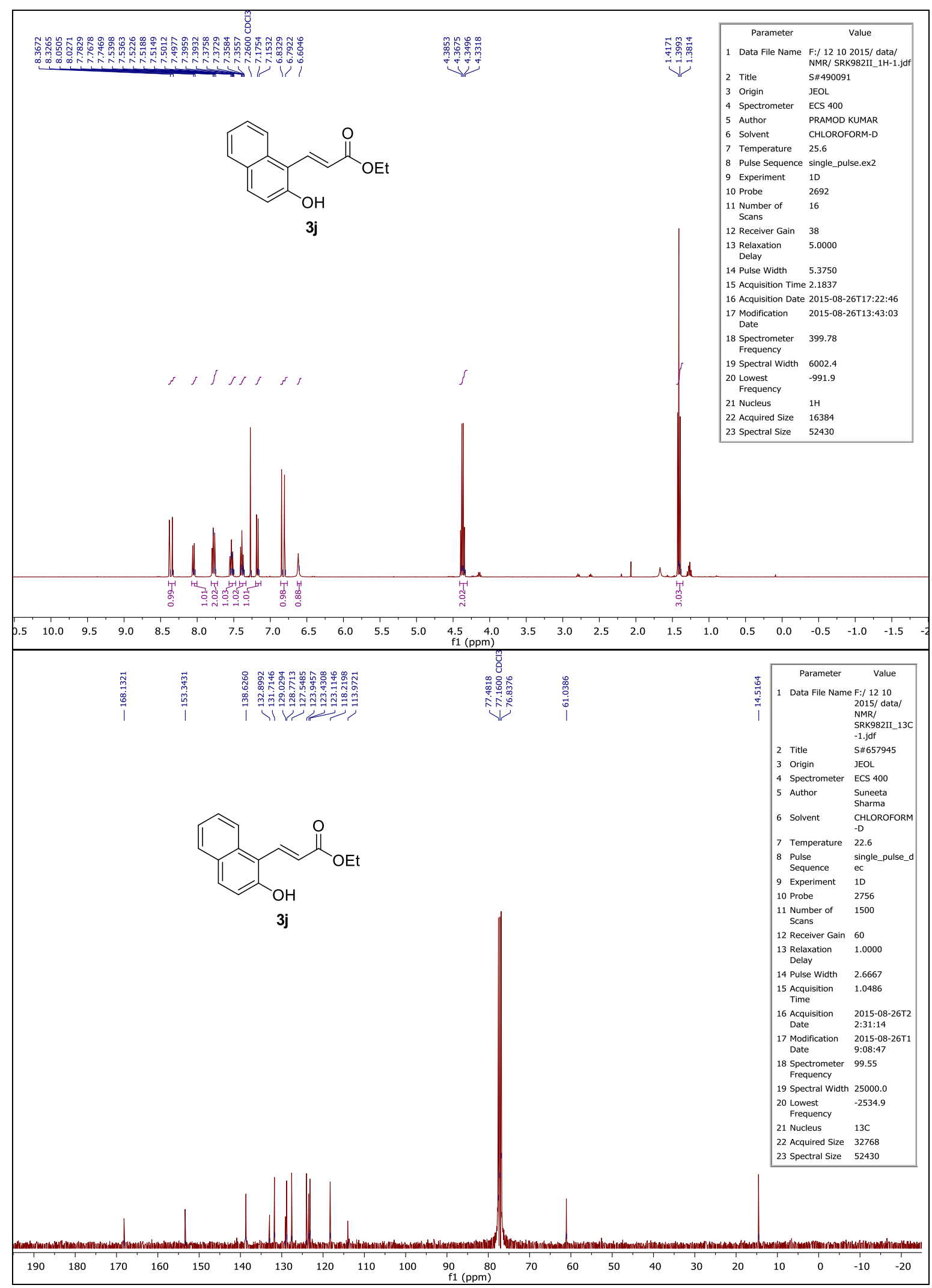

${ }^{1} \mathrm{H}$ and ${ }^{13} \mathrm{C}$ NMR spectrum of (E)-ethyl 3-(2-hydroxynaphthalen-1-yl)acrylate (3j) 


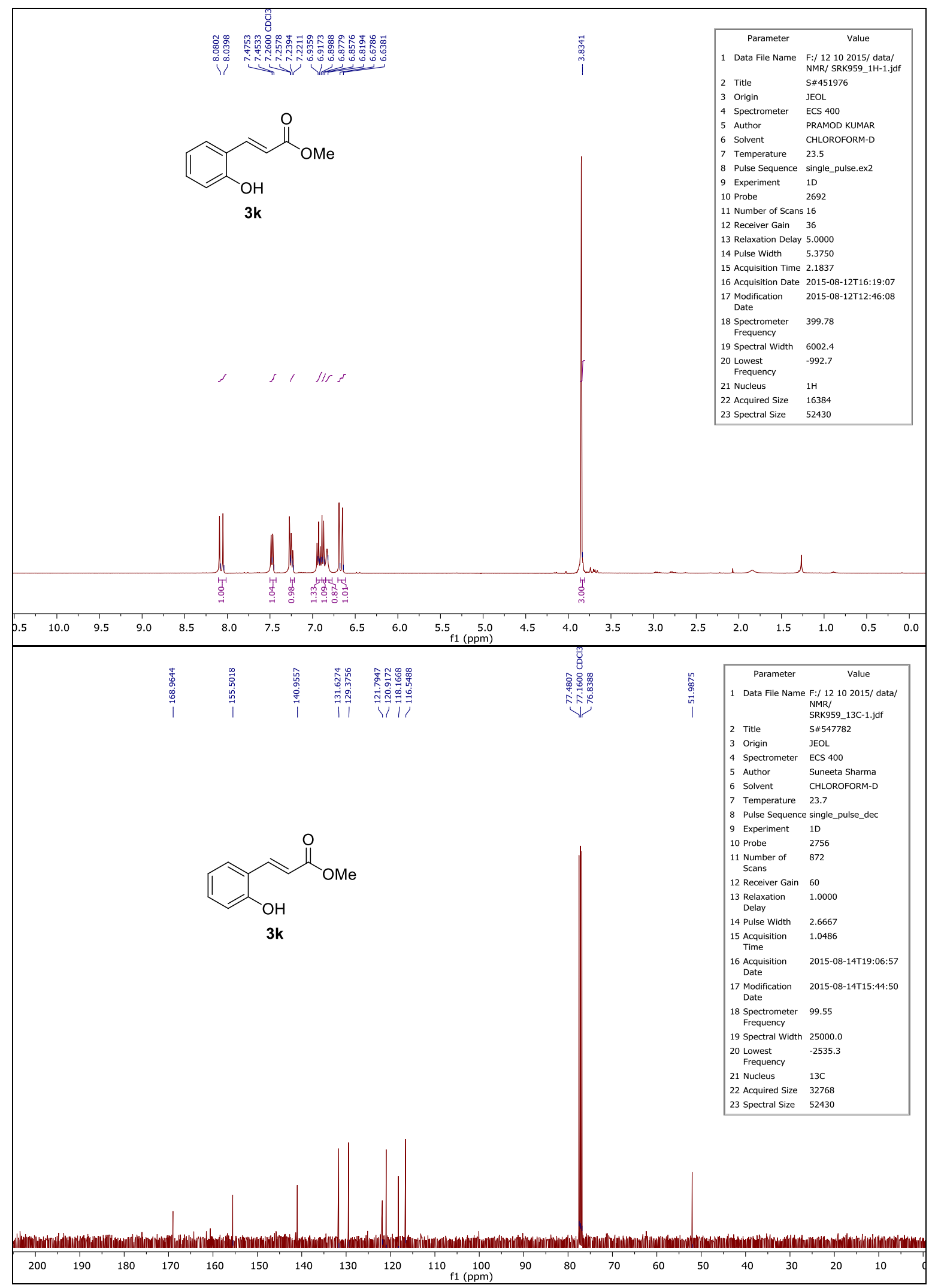

${ }^{1} \mathrm{H}$ and ${ }^{13} \mathrm{C}$ NMR spectrum of (E)-methyl 3-(2-hydroxyphenyl)acrylate (3k) 

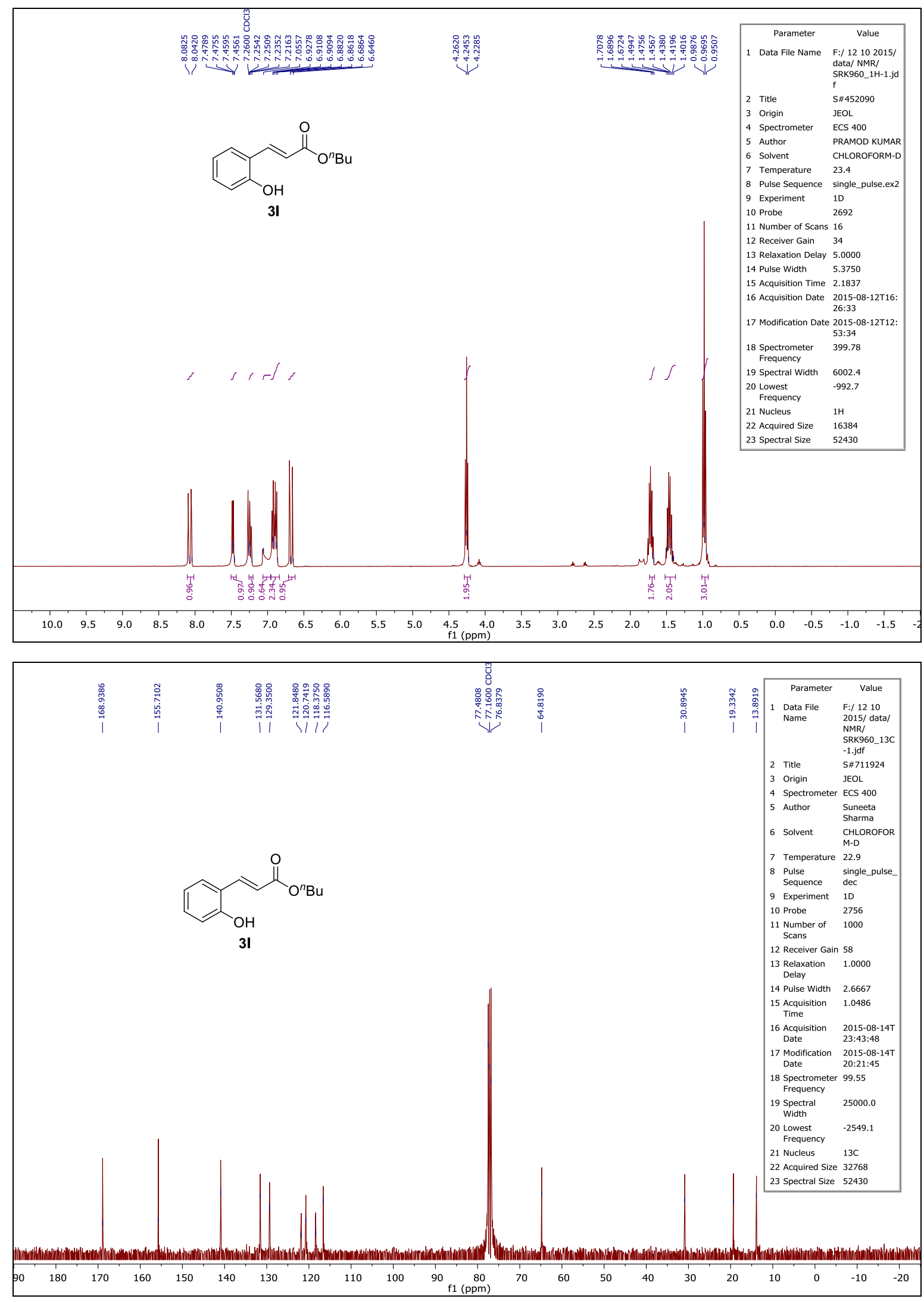

${ }^{1} \mathrm{H}$ and ${ }^{13} \mathrm{C}$ NMR spectrum of (E)-n-butyl 3-(2-hydroxyphenyl)acrylate (3I) 

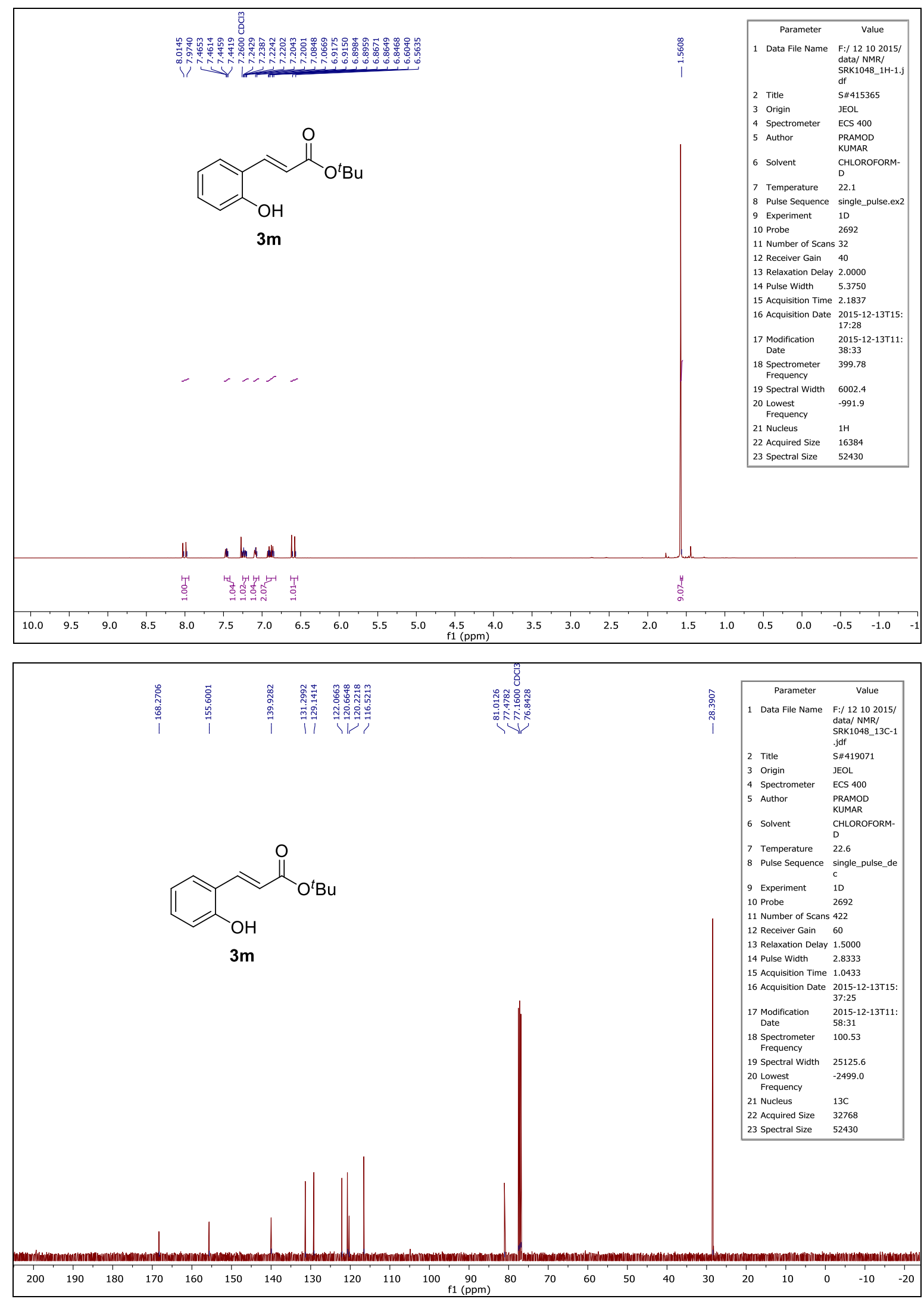

${ }^{1} \mathrm{H}$ and ${ }^{13} \mathrm{C}$ NMR spectrum of (E)-tert-butyl 3-(2-hydroxyphenyl)acrylate (3m) 

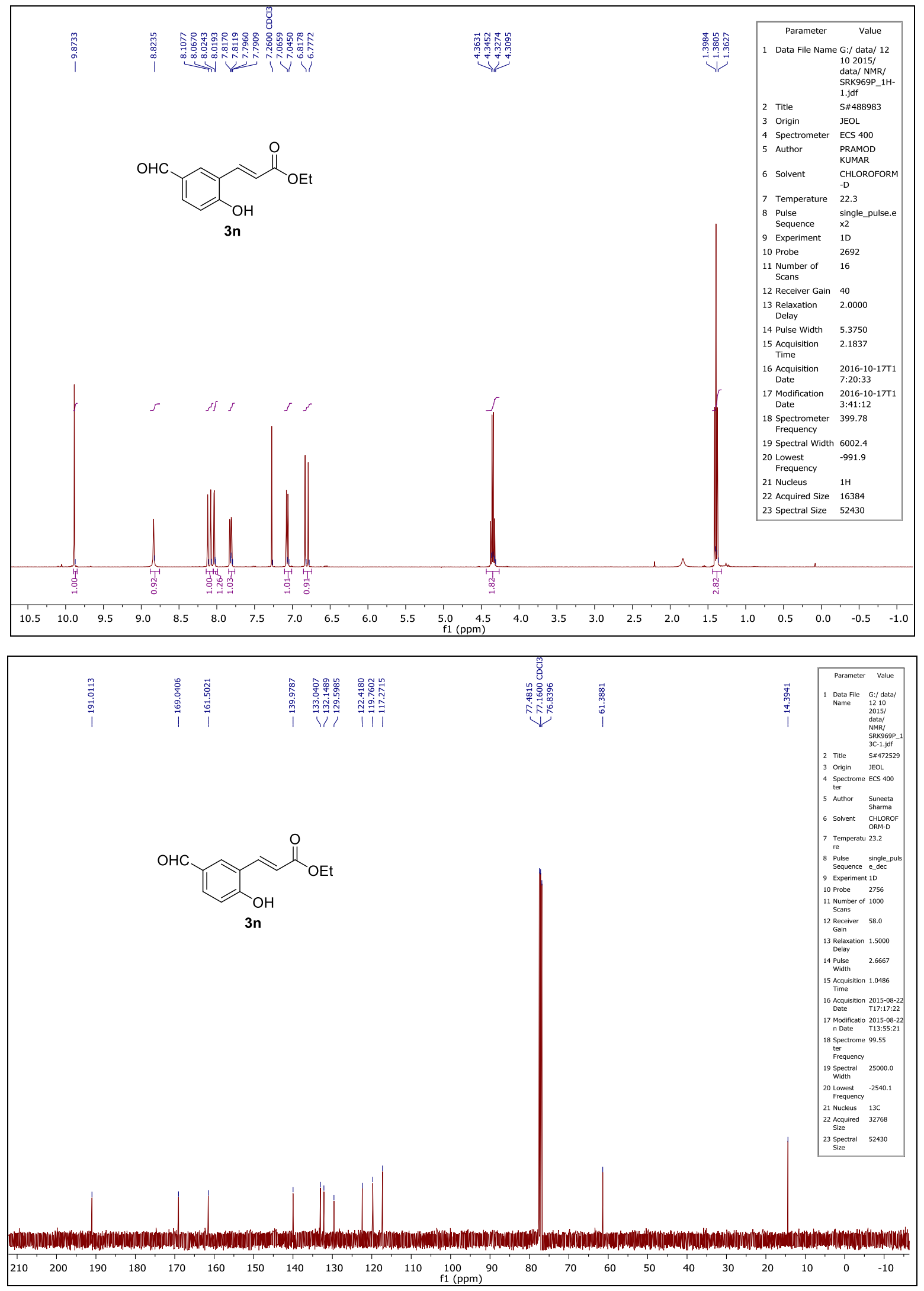

${ }^{1} \mathrm{H}$ and ${ }^{13} \mathrm{C}$ NMR spectrum of (E)-ethyl 3-(5-formyl-2-hydroxyphenyl)acrylate (3n) 

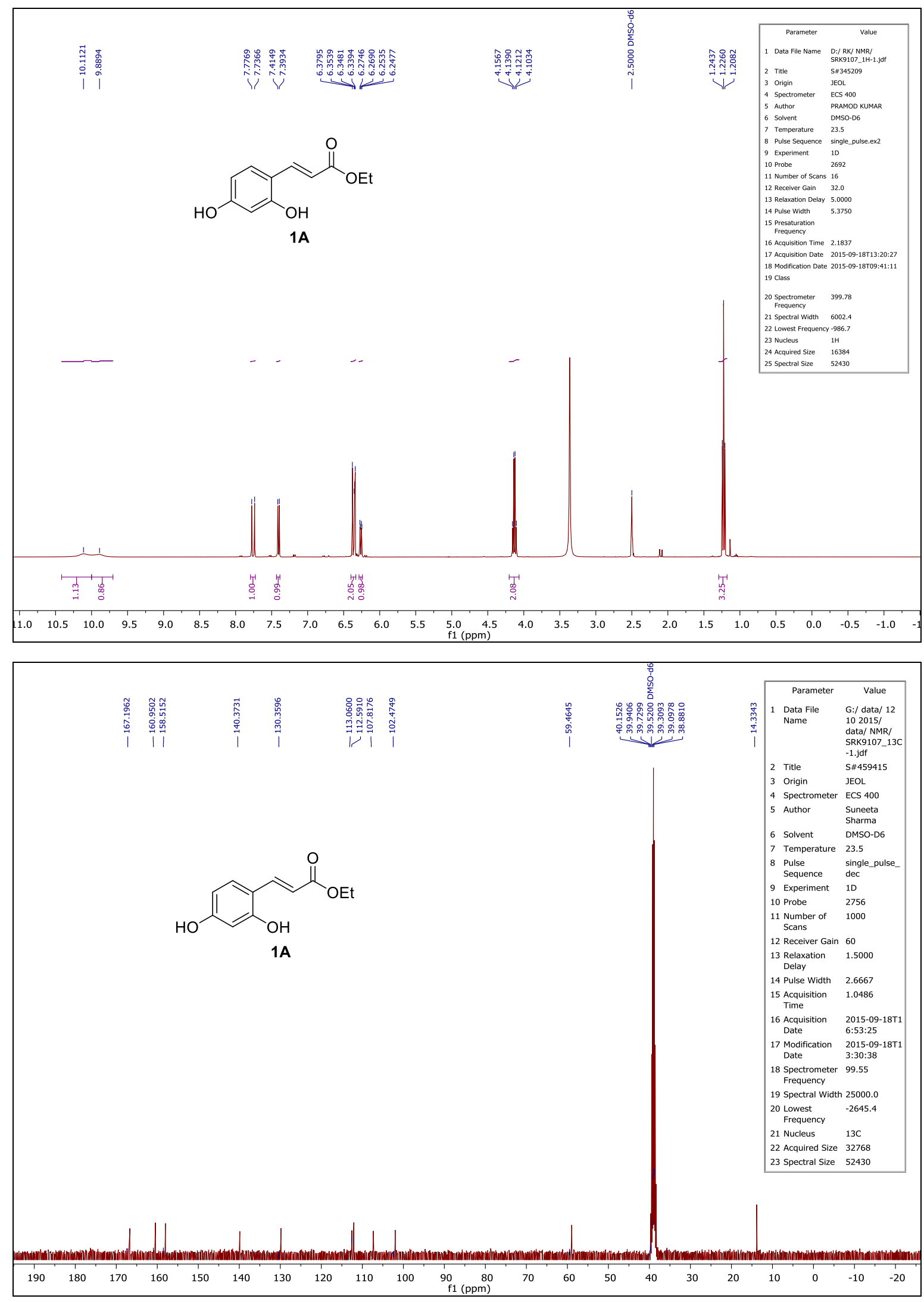

${ }^{1} \mathrm{H}$ and ${ }^{13} \mathrm{C}$ NMR spectrum of (E)-ethyl 3-(2,4-dihydroxyphenyl)acrylate (1A) 

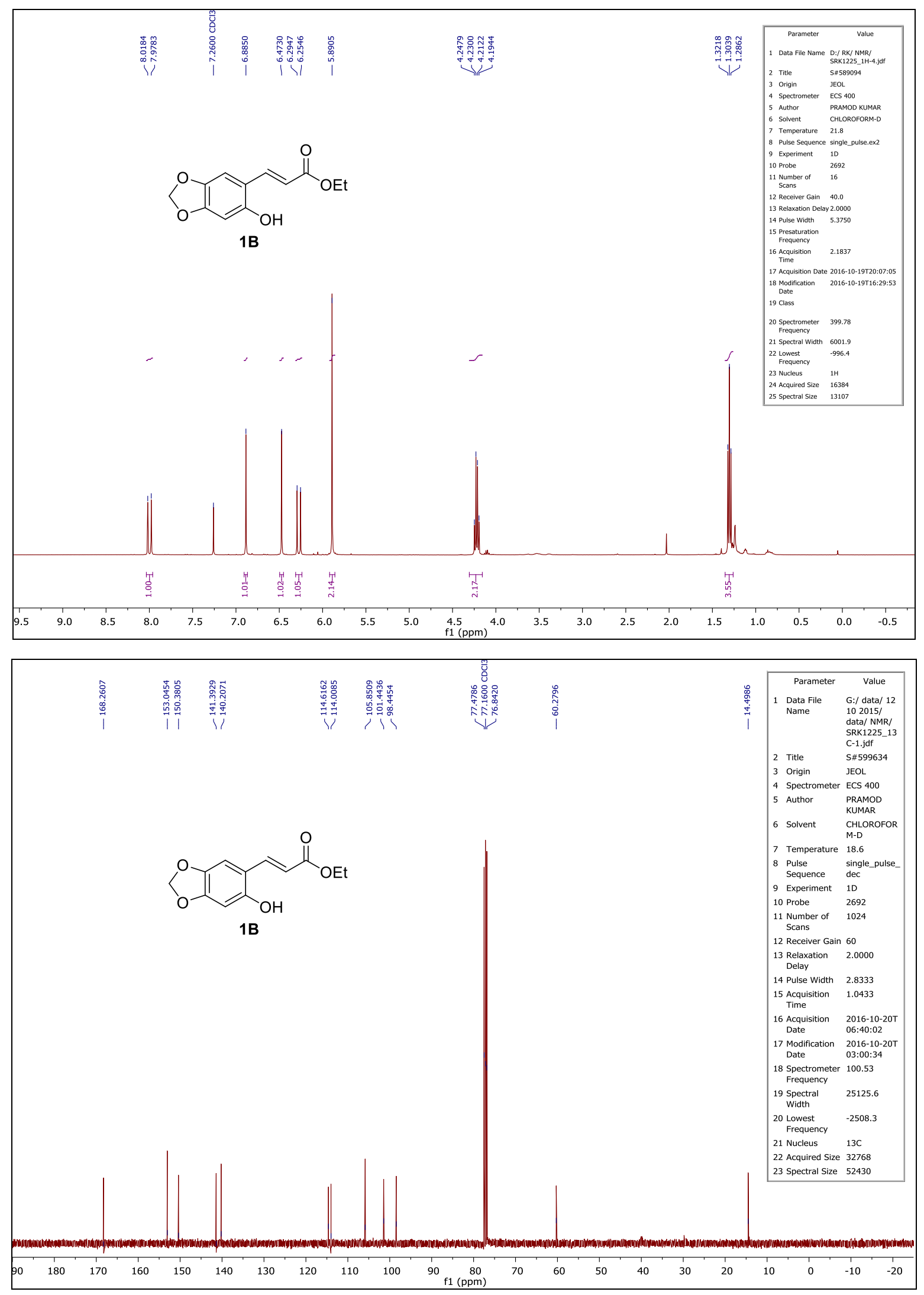

${ }^{1} \mathrm{H}$ and ${ }^{13} \mathrm{C}$ NMR spectrum of (E)-ethyl 3-(6-hydroxybenzo[d][1,3]dioxol-5-yl)acrylate (1B) 


\section{Copies of ${ }^{1} \mathrm{H}$ and ${ }^{13} \mathrm{C}$ NMR spectra of products (4a-4d)}
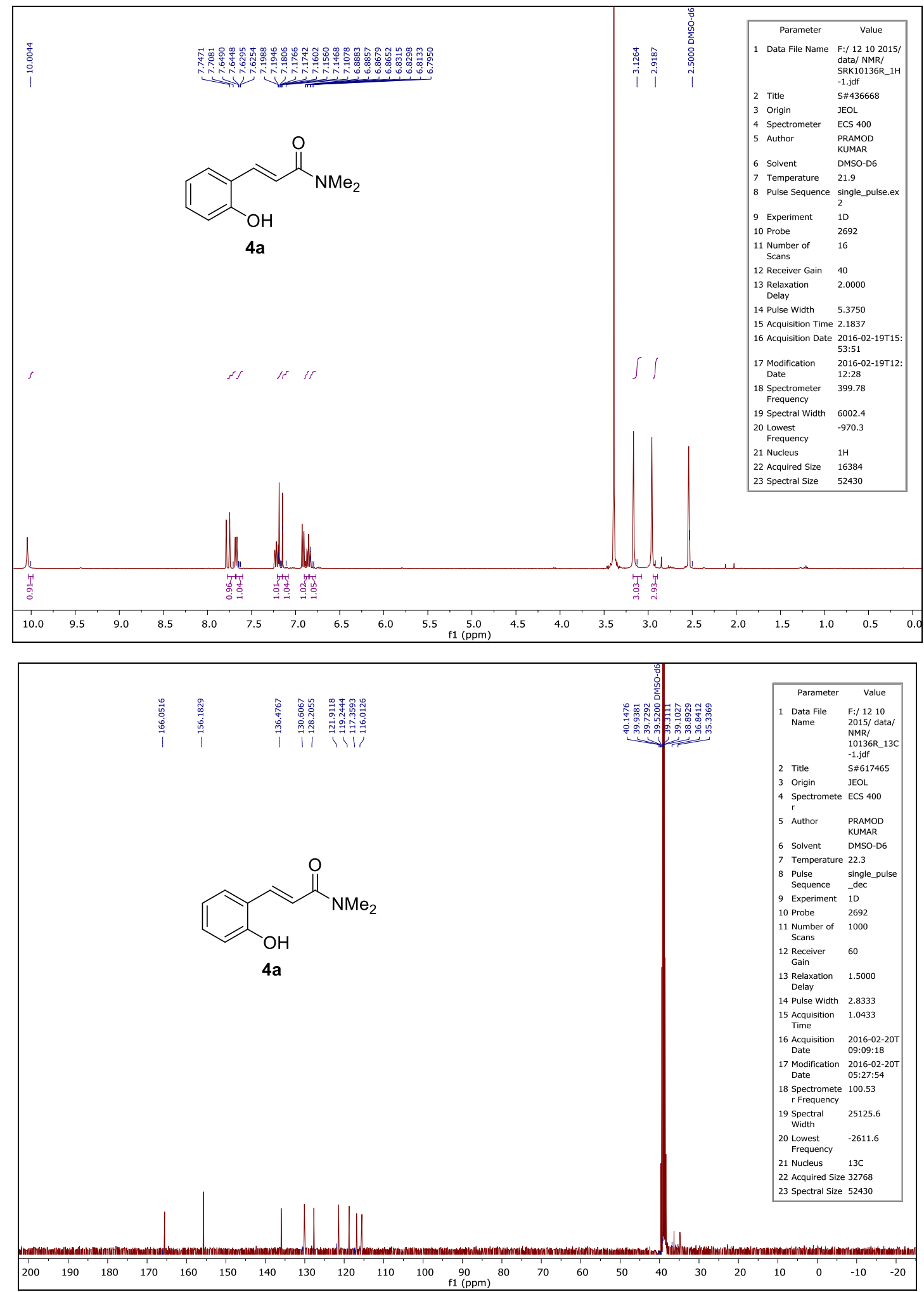

${ }^{1} \mathrm{H}$ and ${ }^{13} \mathrm{C}$ NMR spectrum of (E)-3-(2-hydroxyphenyl)- $N, N$-dimethylacrylamide (4a) 

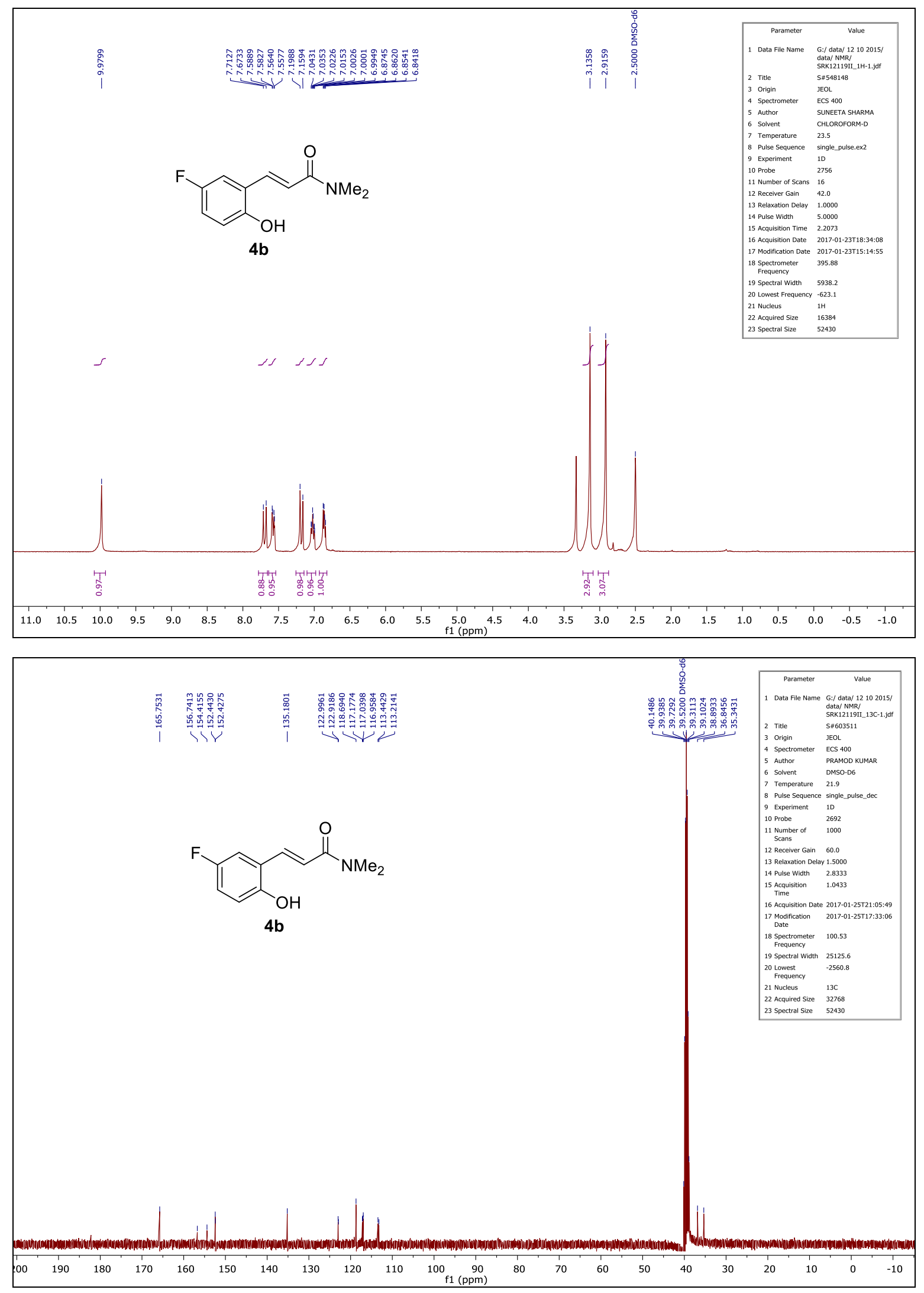

${ }^{1} \mathrm{H}$ and ${ }^{13} \mathrm{C}$ NMR spectrum of (E)-3-(5-fluoro-2-hydroxyphenyl)- $N, N$-dimethylacrylamide (4b) 

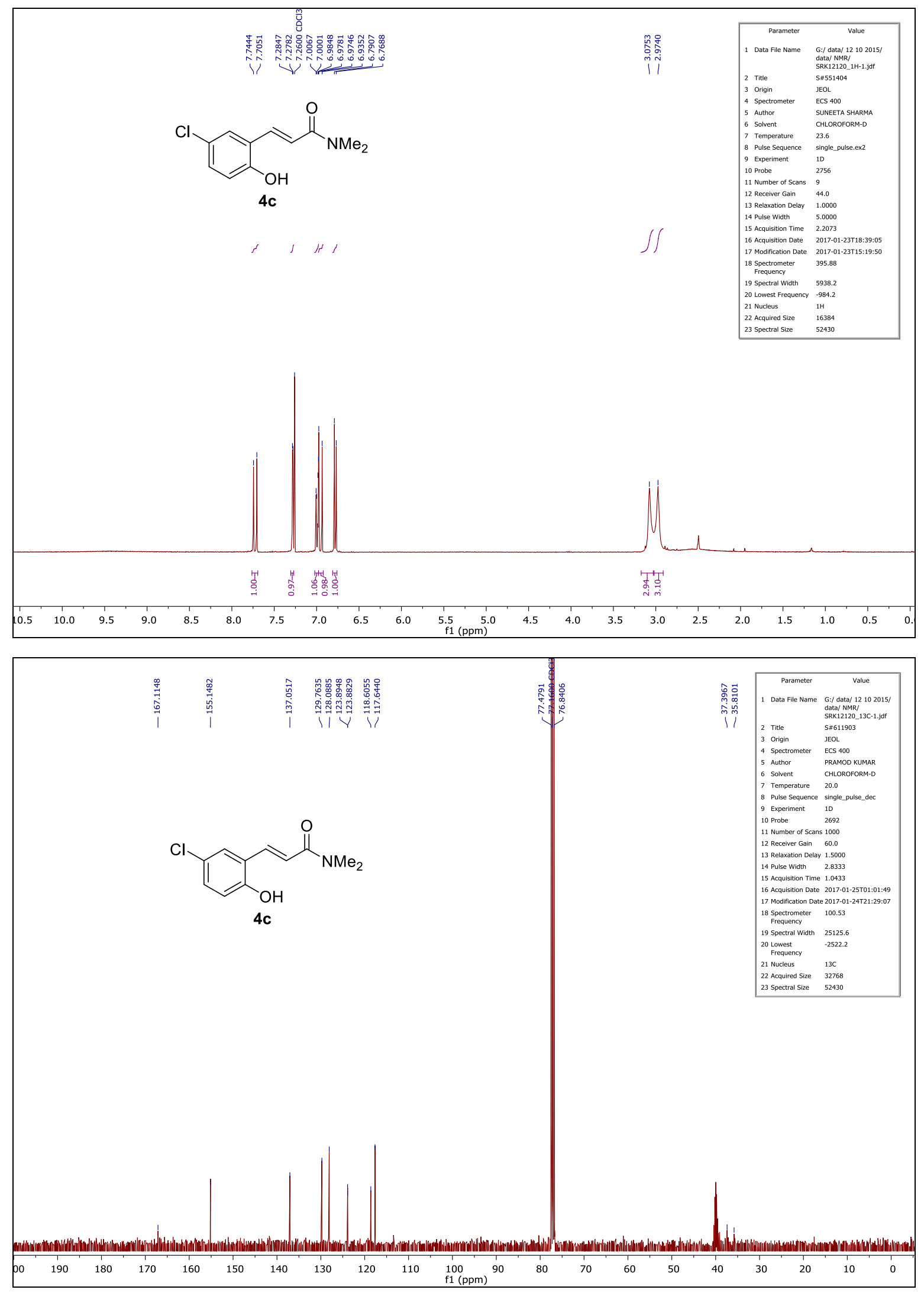

${ }^{1} \mathrm{H}$ and ${ }^{13} \mathrm{C}$ NMR spectrum of (E)-3-(5-chloro-2-hydroxyphenyl)- $N, N$-dimethylacrylamide (4c) 

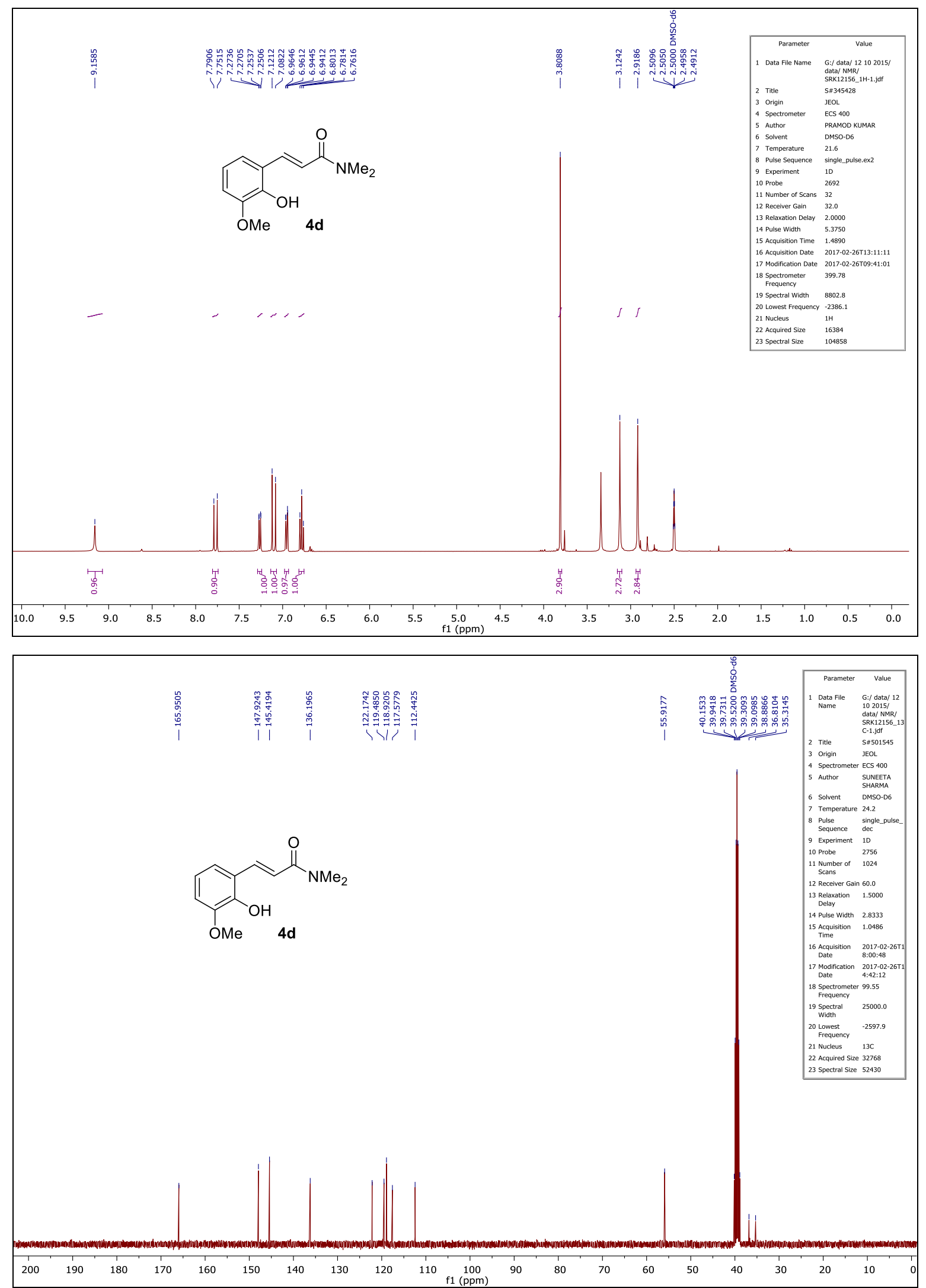

${ }^{1} \mathrm{H}$ and ${ }^{13} \mathrm{C}$ NMR spectrum of (E)-3-(2-hydroxy-3-methoxyphenyl)- $N, N$-dimethylacrylamide (4d) 
3. Copies of HRMS spectra of products (3a-3n, 1A, 1B and 4a-4d)

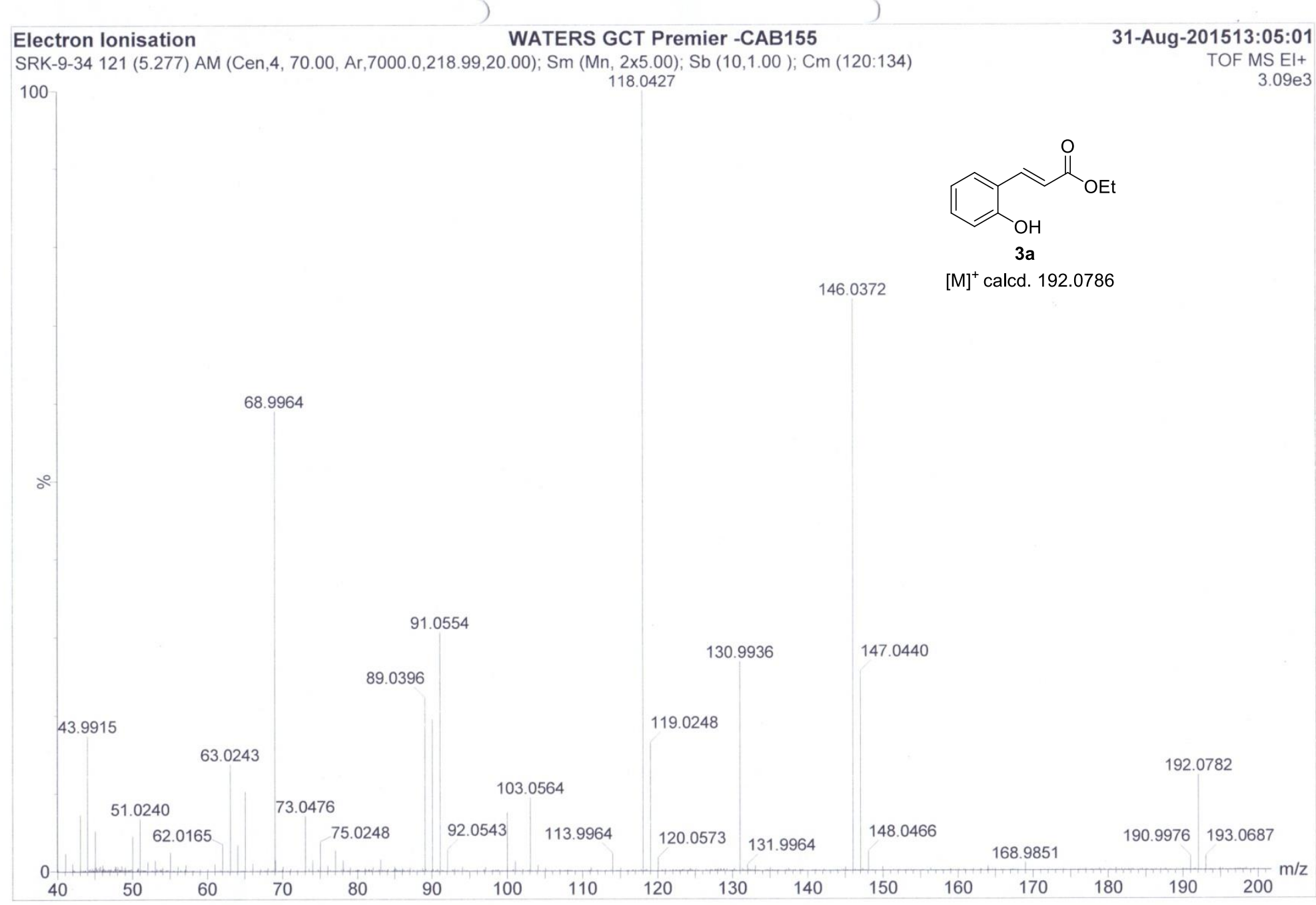

EI(HRMS) spectrum of (E)-ethyl 3-(2-hydroxyphenyl)acrylate (3a) 


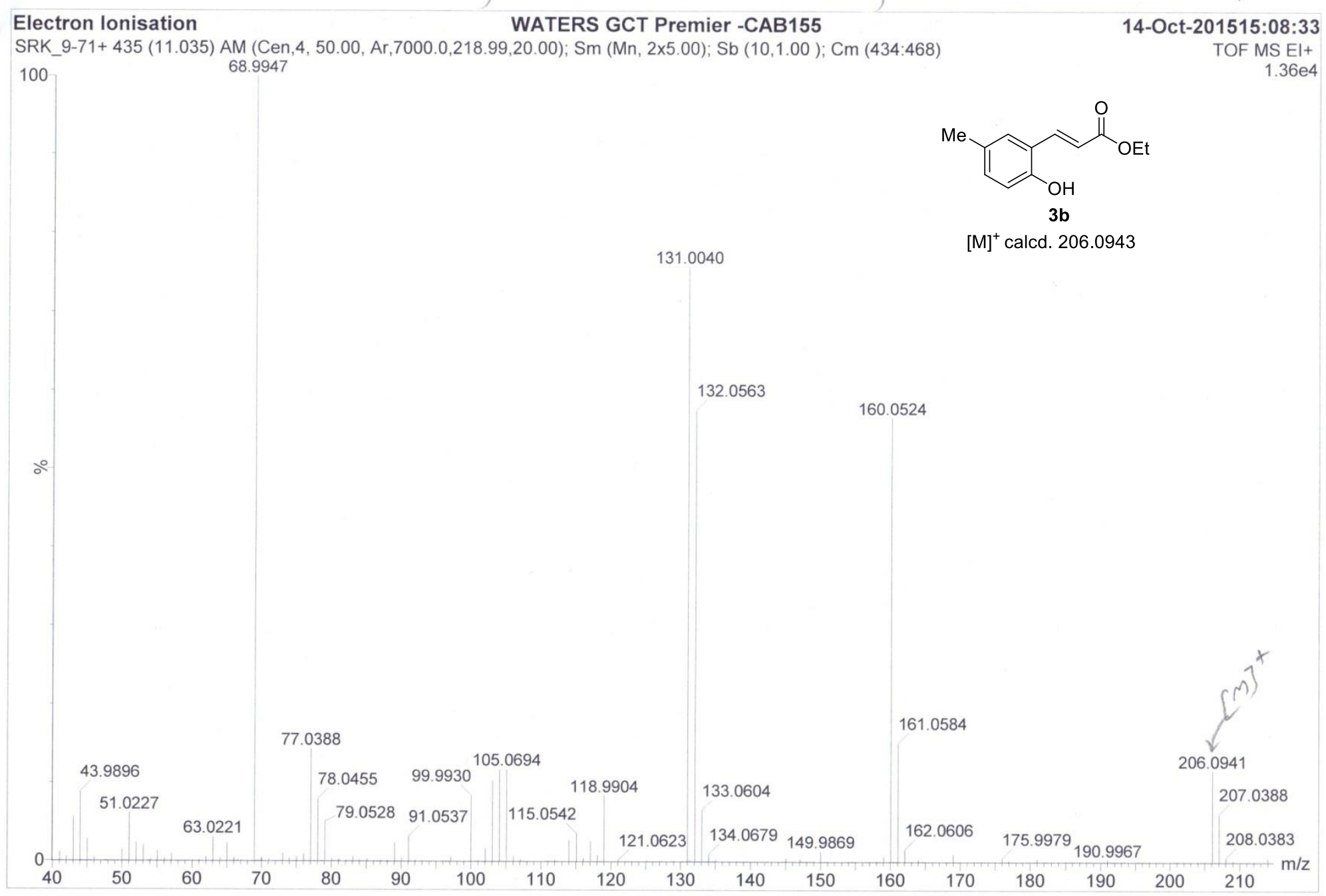

EI(HRMS) spectrum of (E)-ethyl 3-(2-hydroxy-5-methylphenyl)acrylate (3b) 


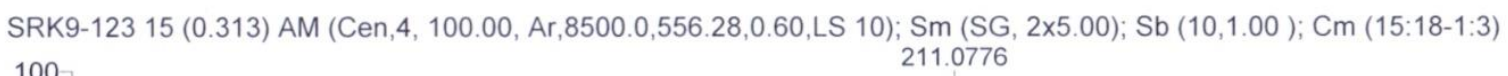

211.0776

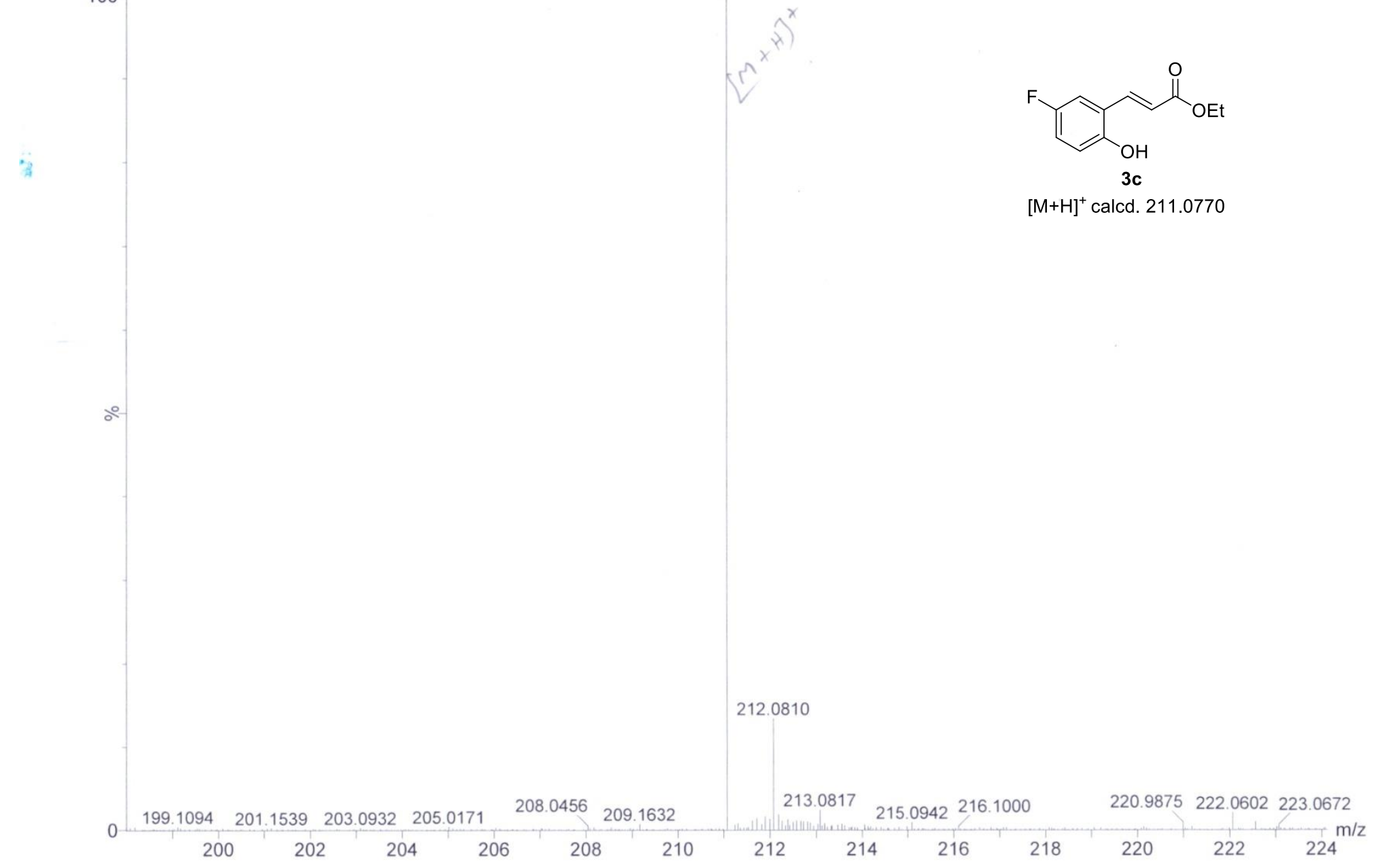

ESI(HRMS) spectrum of (E)-ethyl 3-(5-fluoro-2-hydroxyphenyl)acrylate (3c) 


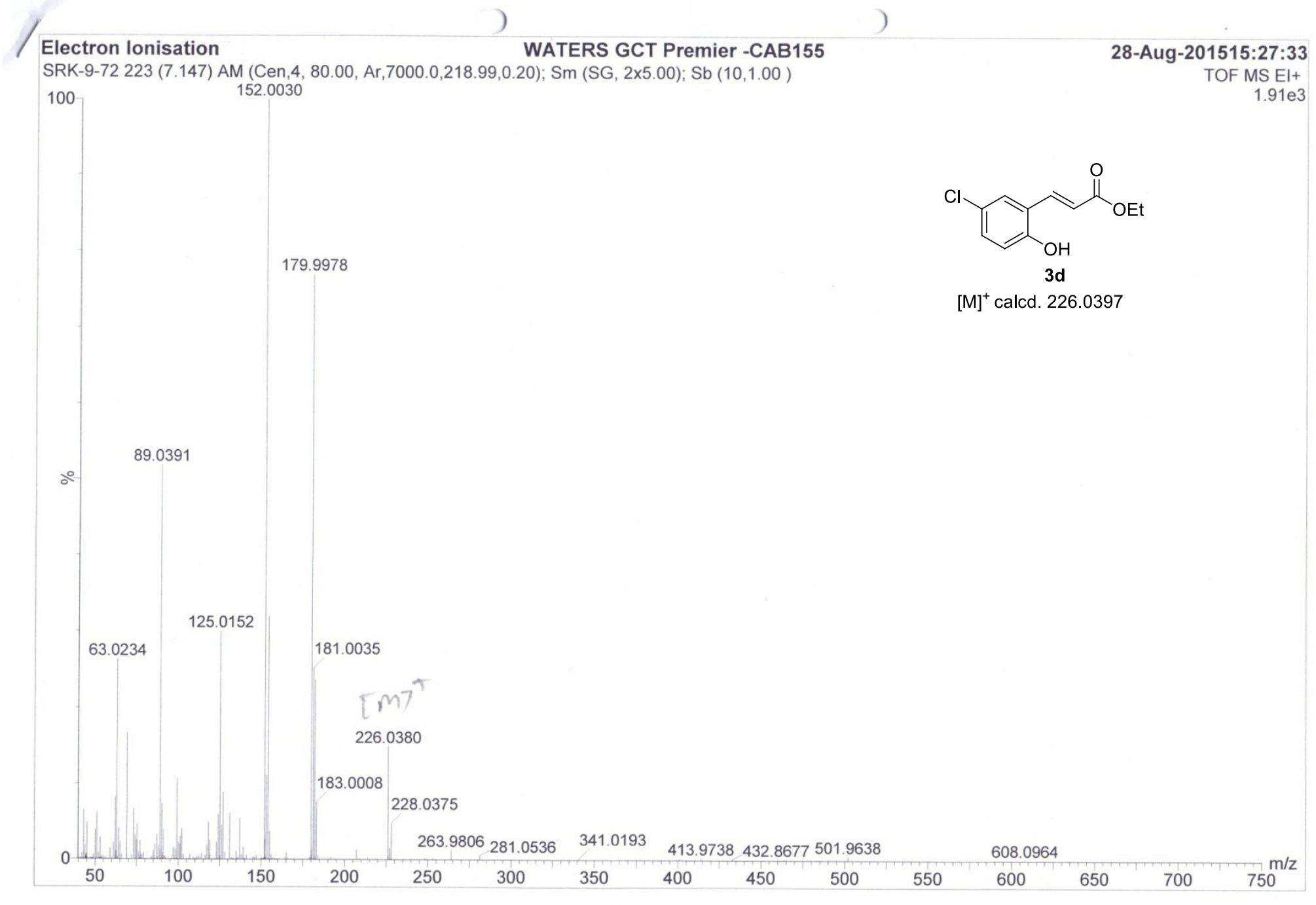

EI(HRMS) spectrum of (E)-ethyl 3-(5-chloro-2-hydroxyphenyl)acrylate (3d) 
SRK9-81 10 (0.221) AM (Cen,4, 100.00, Ar,8500.0,556.28,0.70,LS 10); Sm (SG, 2x5.00); Sb (10,1.00); Cm (9:10-72:81)

100 $270.9977 \quad 272.9951$

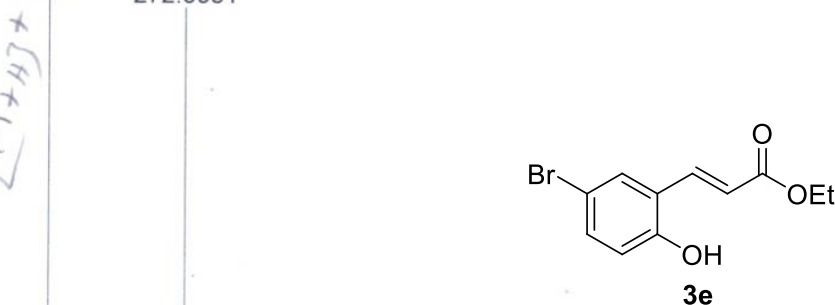

$[\mathrm{M}+\mathrm{H}]^{+}$calcd. 270.9970

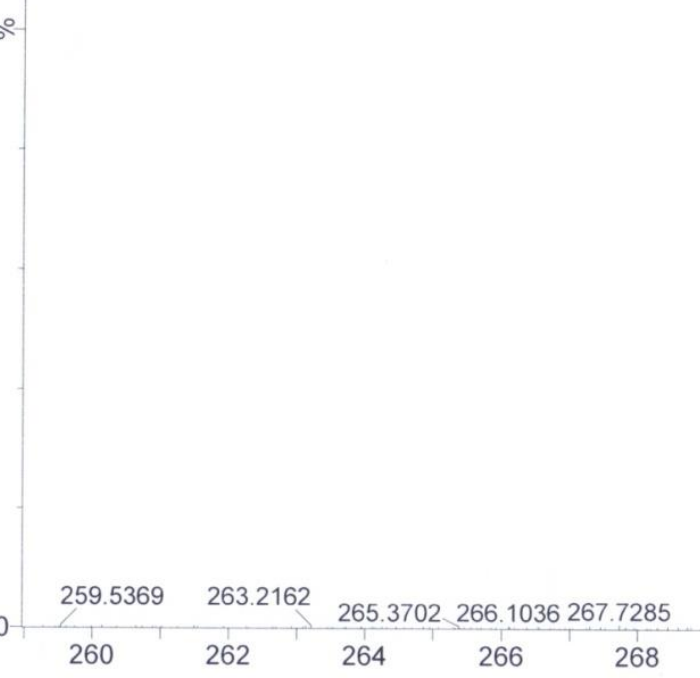

ESI(HRMS) spectrum of (E)-ethyl 3-(5-bromo-2-hydroxyphenyl)acrylate (3e) 


\section{Electrospray ionisation -MS}

SRK9-117-I 12 (0.258) AM (Cen,4, 100.00, Ar,8500.0,556.28,0.70,LS 10); Sm (SG, 2x5.00); Sb (10,1.00); Cm (11:13-1:3)

1: TOF MS

$7.96 \mathrm{e} 3$
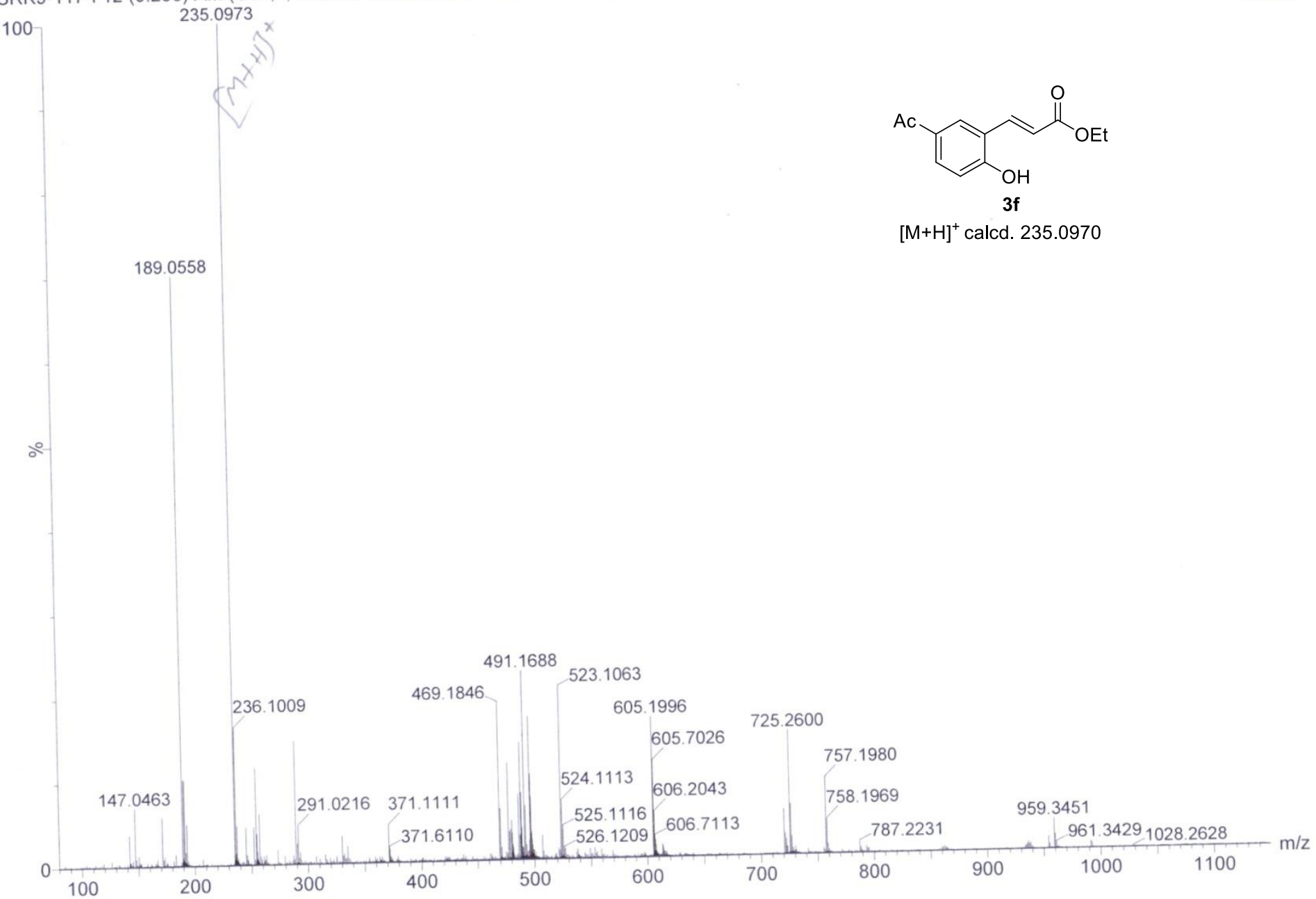

ESI(HRMS) spectrum of (E)-ethyl 3-(5-acetyl-2-hydroxyphenyl)acrylate (3f) 


\section{Electrospray ionisation -MS}

SRK9-83 16 (0.332) AM (Cen,4, 100.00, Ar,8500.0,556.28,0.70,LS 10); Sm (SG, 1x5.00); Sb (10,10.00); Cm (16:18-98:104)

100 223.0970

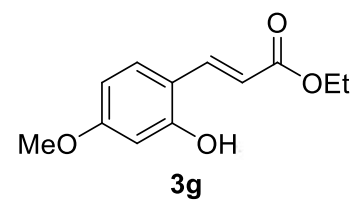

$[\mathrm{M}+\mathrm{H}]^{+}$calcd. 223.0970

\subsection{5}

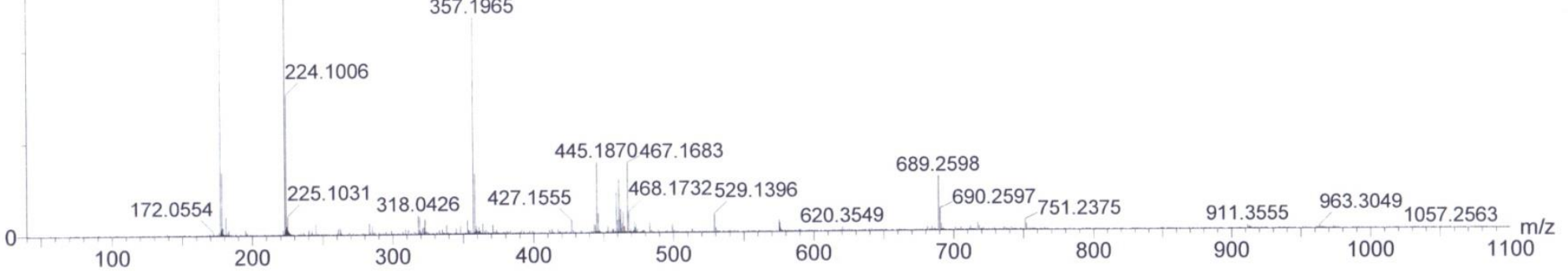

ESI(HRMS) spectrum of (E)-ethyl 3-(2-hydroxy-4-methoxyphenyl)acrylate (3g) 


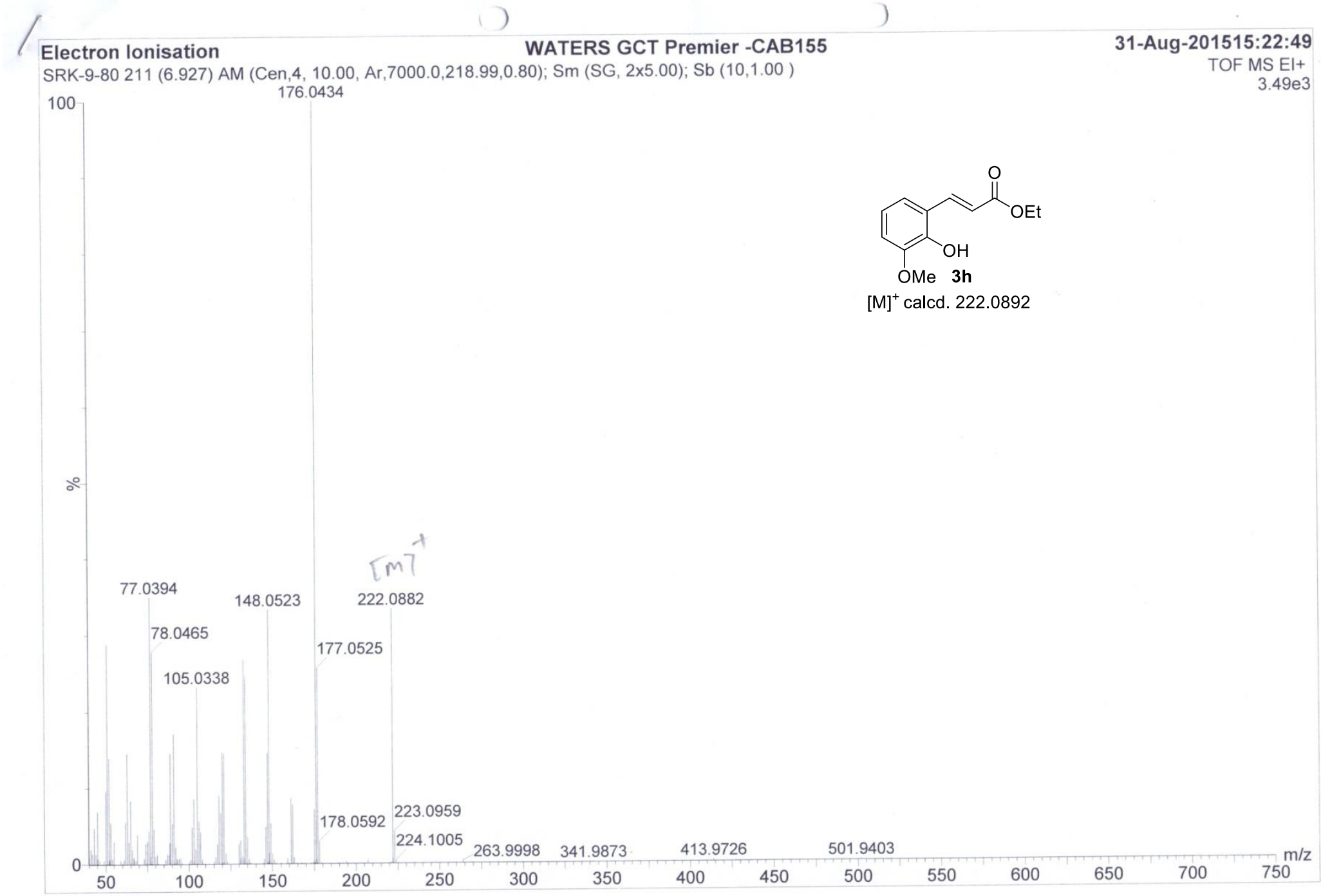

EI(HRMS) spectrum of (E)-ethyl 3-(2-hydroxy-3-methoxyphenyl)acrylate (3h) 


\section{Electrospray ionisation -MS}

SRK9-87 15 (0.314) AM (Cen,4, 100.00, Ar,8500.0,556.28,0.35,LS 10); Sm (SG, 1x5.00); Sb (10,10.00); Cm (15:19-67:72) 283.1183

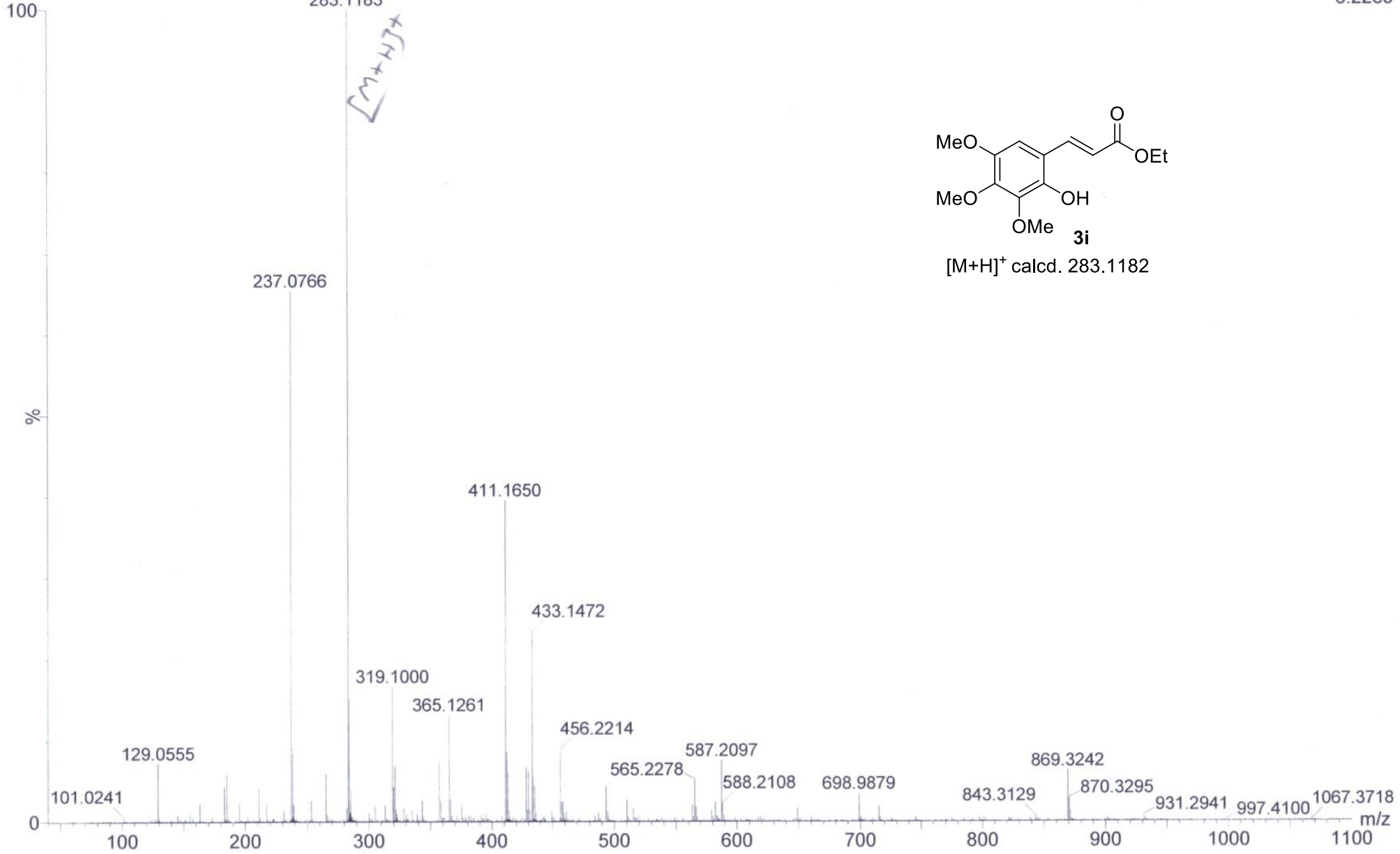

ESI(HRMS) spectrum of (E)-ethyl 3-(2-hydroxy-3,4,5-trimethoxyphenyl)acrylate (3i) 


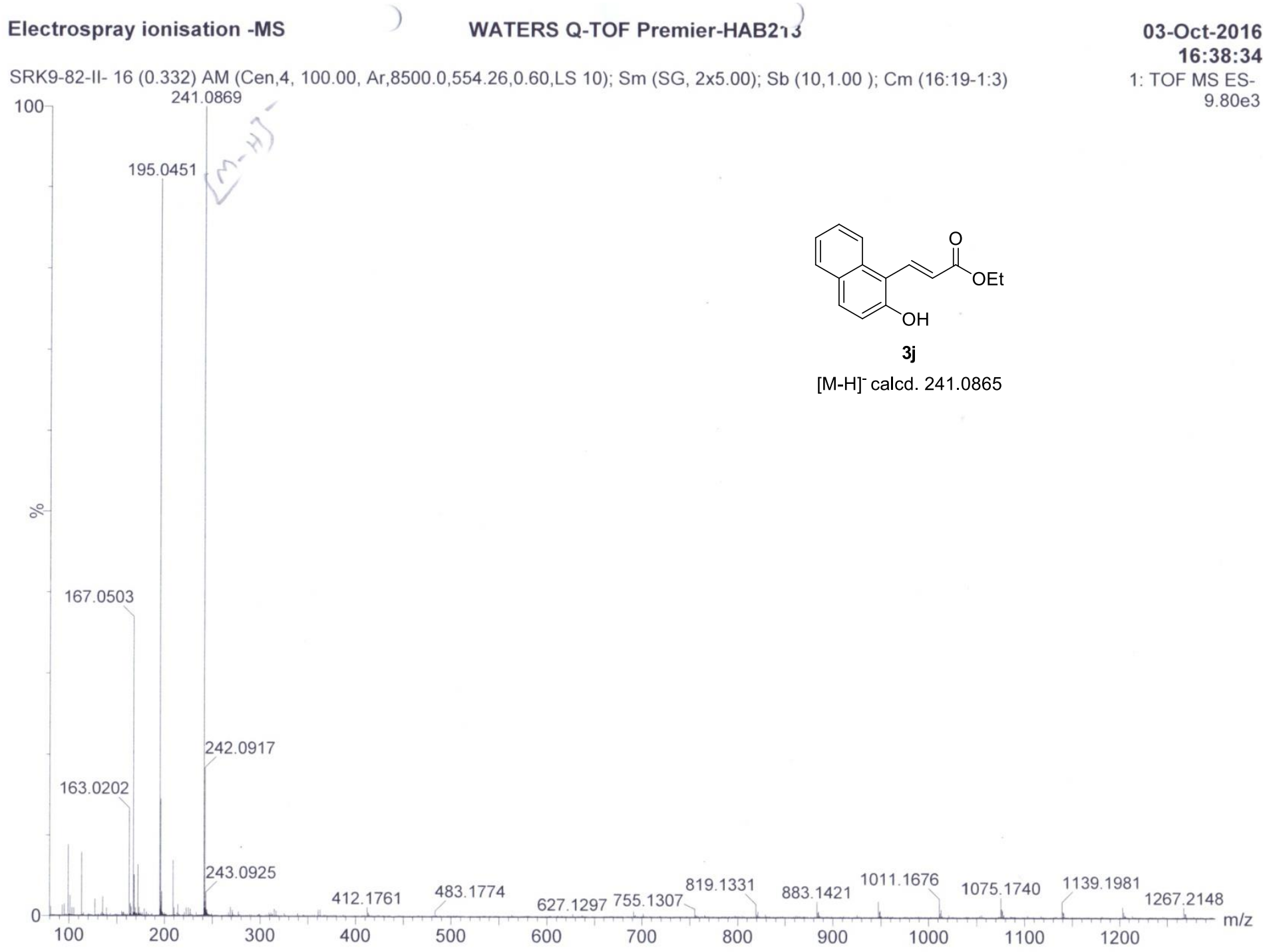

ESI(HRMS) spectrum of (E)-ethyl 3-(2-hydroxynaphthalen-1-yl)acrylate (3j) 


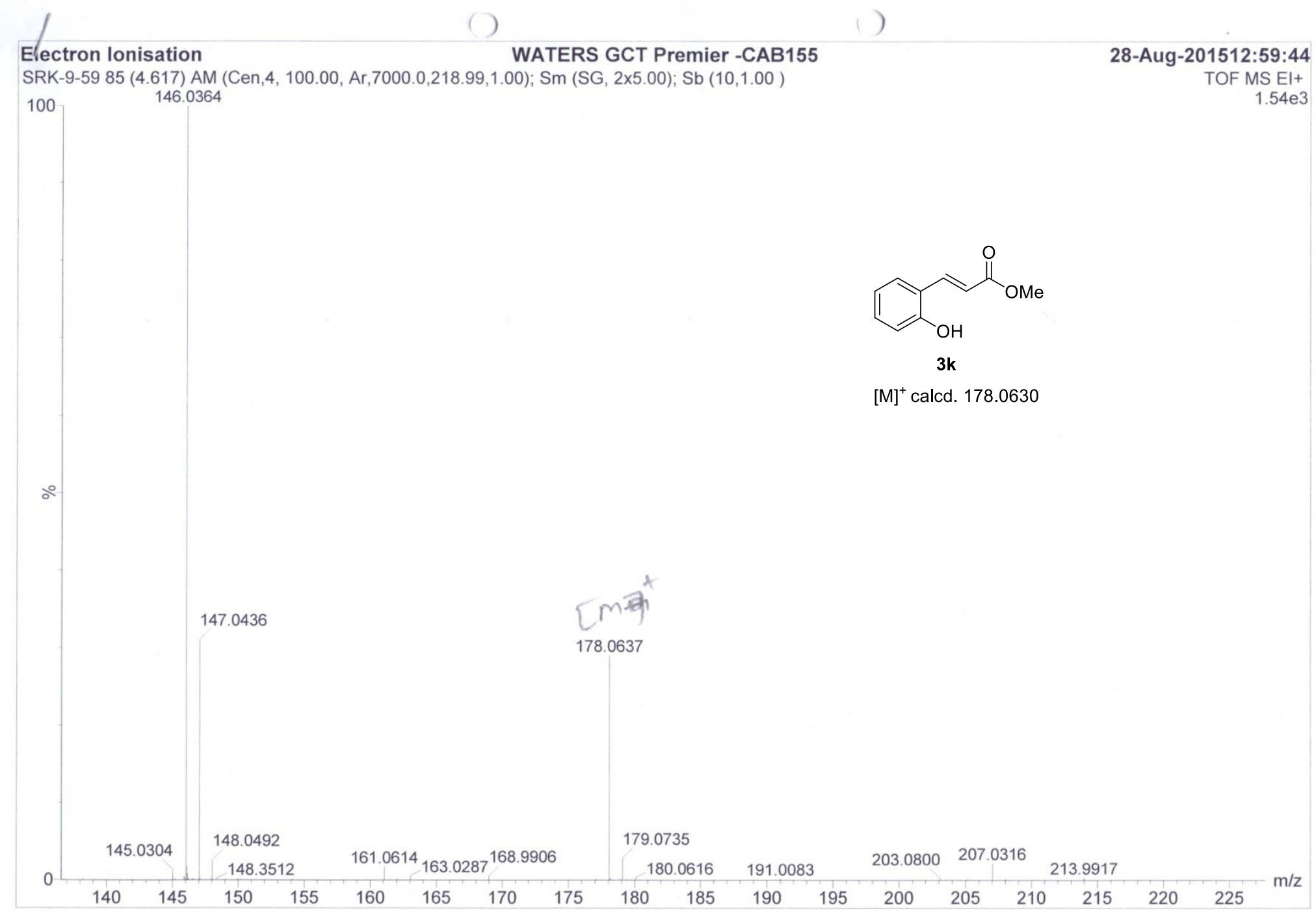

EI(HRMS) spectrum of (E)-methyl 3-(2-hydroxyphenyl)acrylate (3k) 


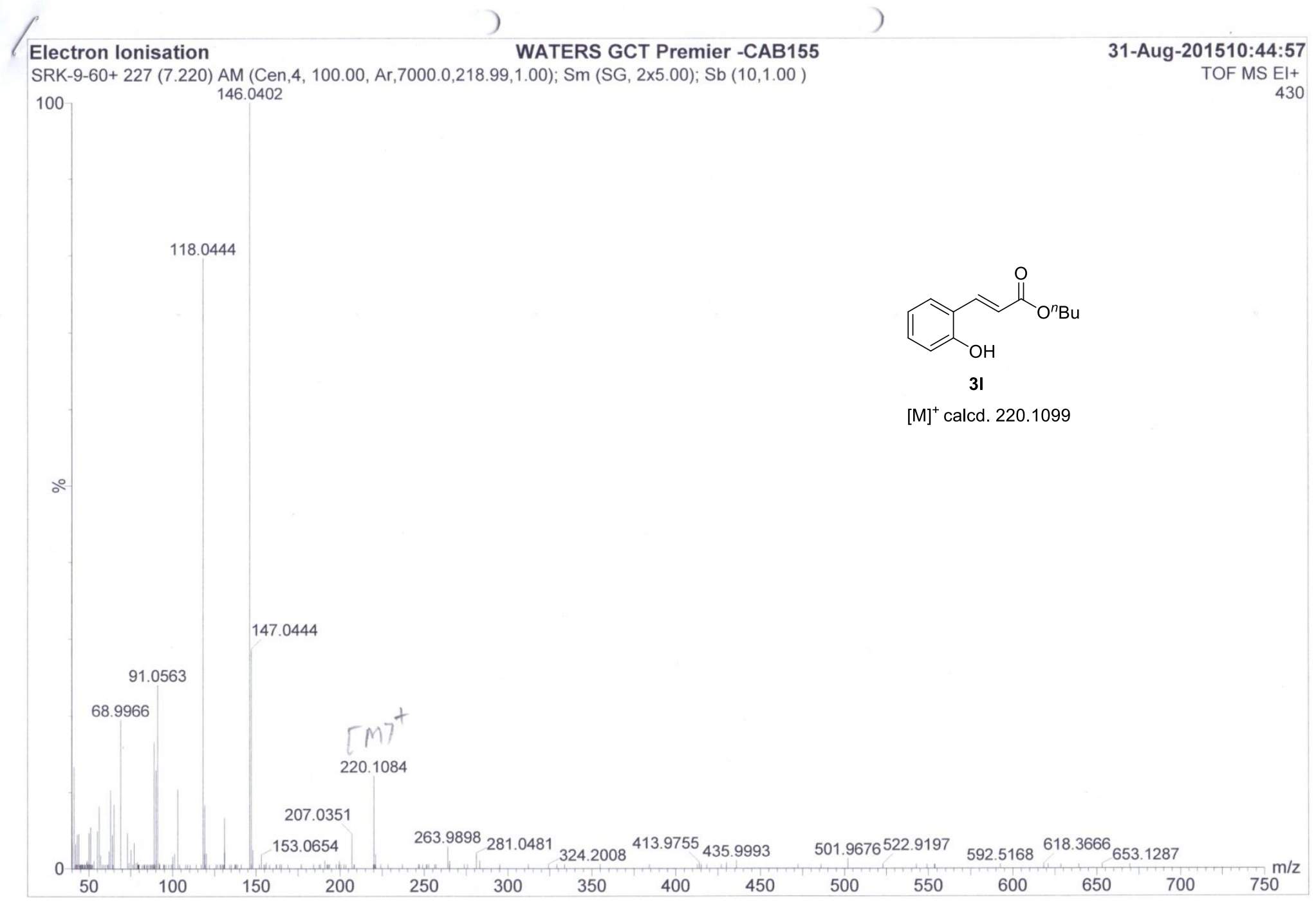

EI(HRMS) spectrum of (E)-n-butyl 3-(2-hydroxyphenyl)acrylate (3I) 
SRK10-48 14 (0.296) AM (Cen,4, 100.00, Ar,8500.0,556.28,0.70,LS 10); Sm (SG, 2x5.00); Sb (10,1.00); Cm (12:16-1:3) 100 243.0998

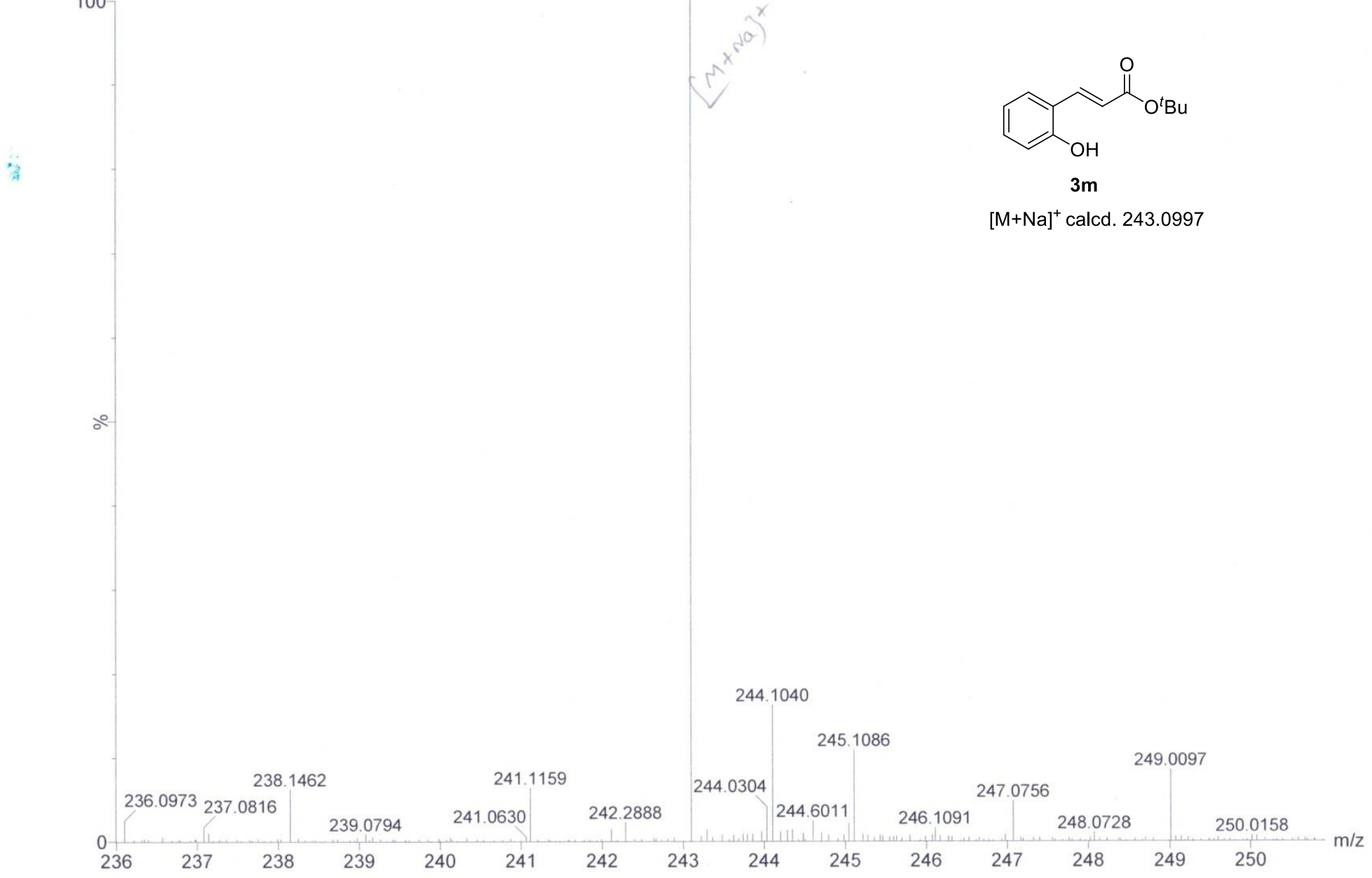

ESI(HRMS) spectrum of (E)-tert-butyl 3-(2-hydroxyphenyl)acrylate (3m) 


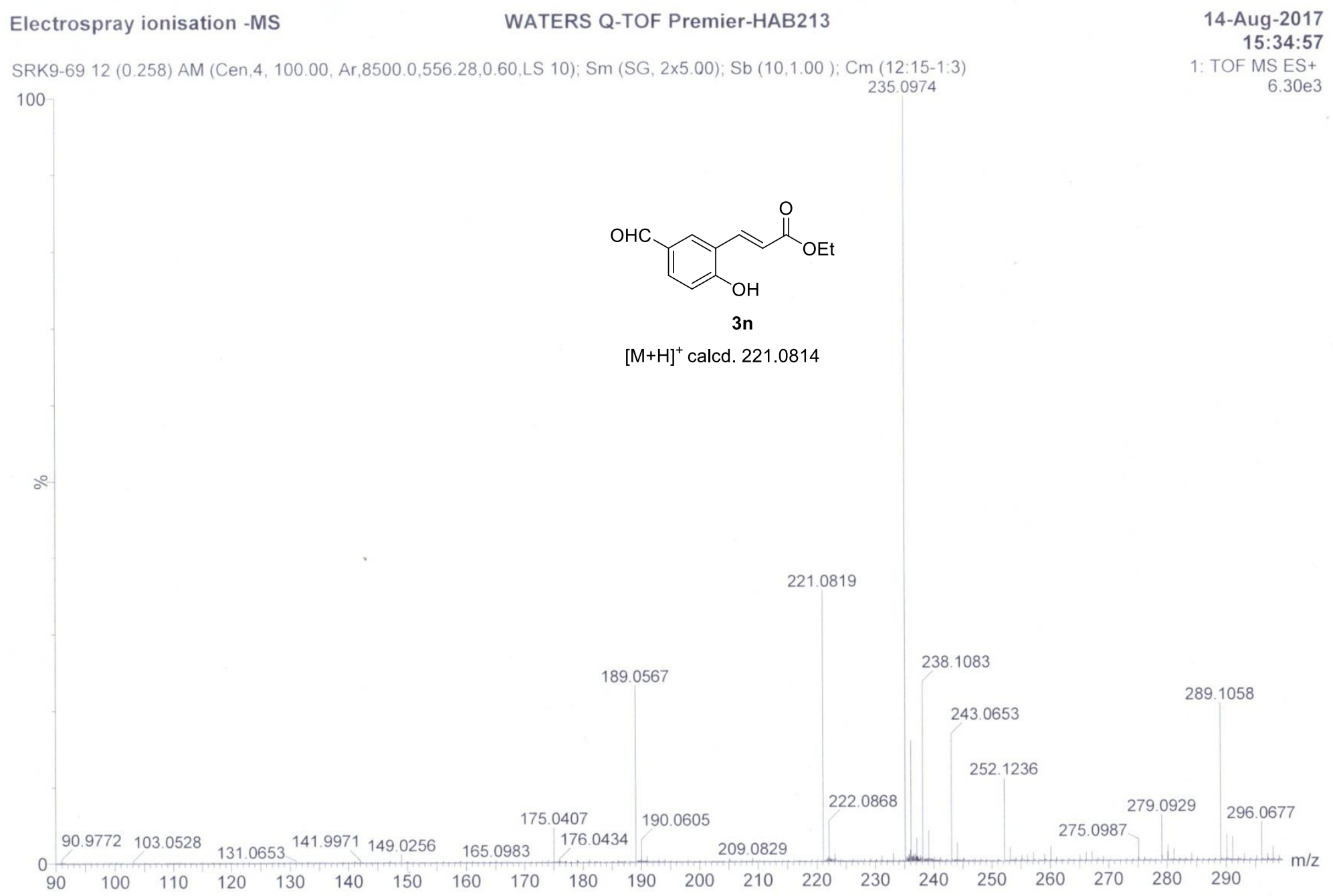

ESI(HRMS) spectrum of (E)-ethyl 3-(5-formyl-2-hydroxyphenyl)acrylate (3n) 
SRK9-107- 17 (0.3F9) AM (Cen,4, 100.00, Ar,10000.C.554.26,0.70,LS 10); Sm (SG, 1x5.00); Sb (15,10.00); Cm (17:20-1:3) 207.0642

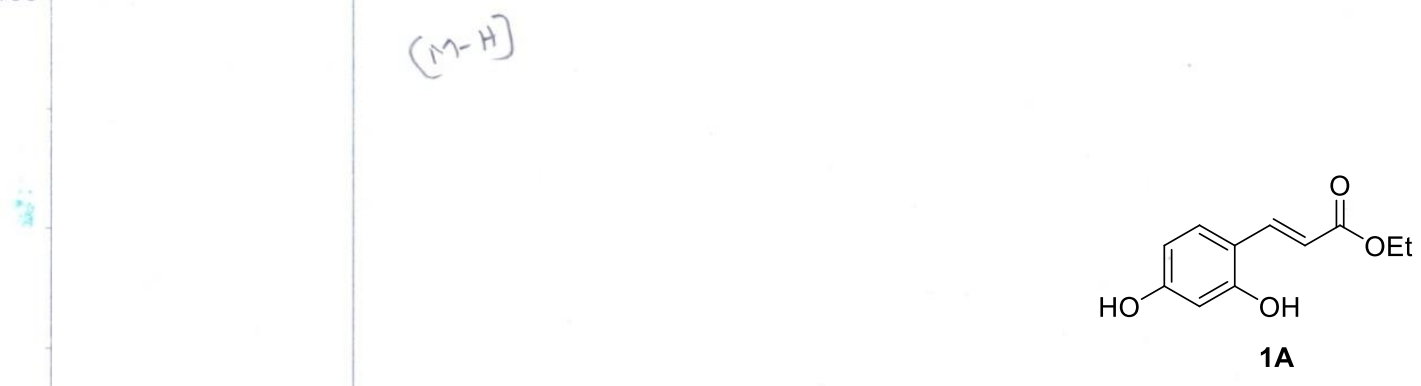

$[\mathrm{M}-\mathrm{H}]^{-}$calcd. 207.0657

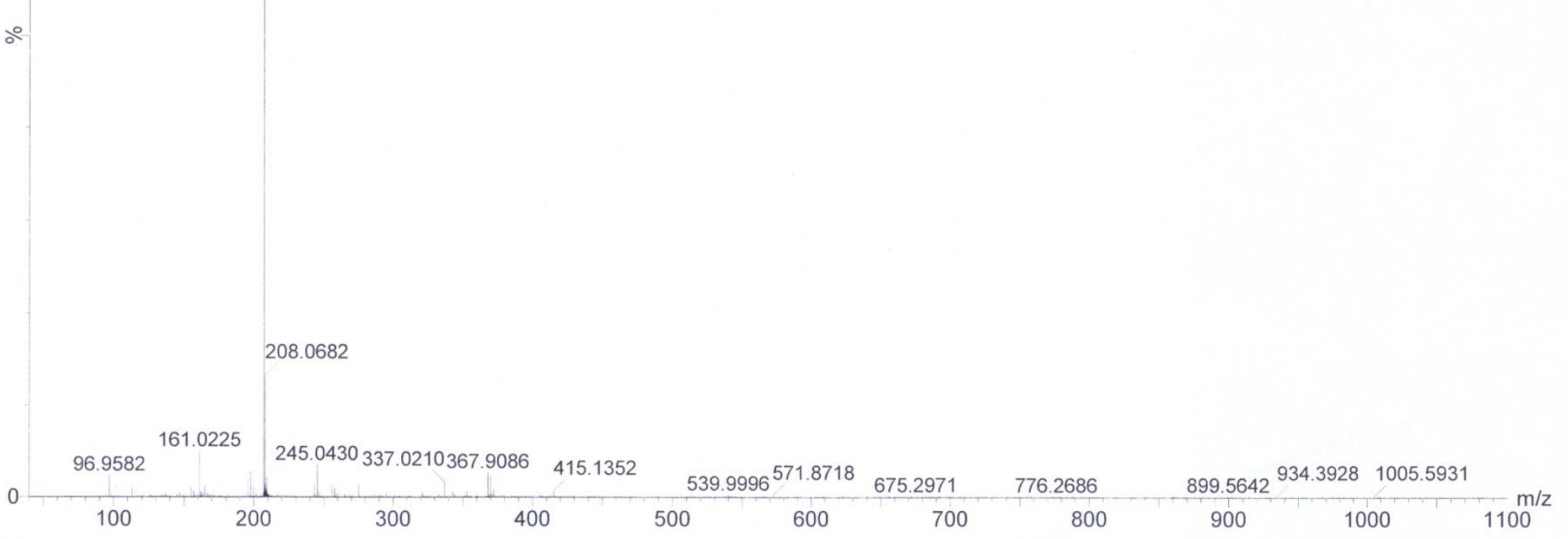

ESI(HRMS) spectrum of (E)-ethyl 3-(2,4-dihydroxyphenyl)acrylate (1A) 


\section{Electrospray ionisation -MS}

WATERS Q-TOF Premier-HAB213

19-Oct-2016

16:14:09

SRK9-148 14 (0.295) AM (Cen,4, 100.00, Ar,8500.0,556.28,0.75,LS 10); Sm (SG, 2x5.00); Sb (10,1.00); Cm (14:15-1:3)

100 237.0769

3.963

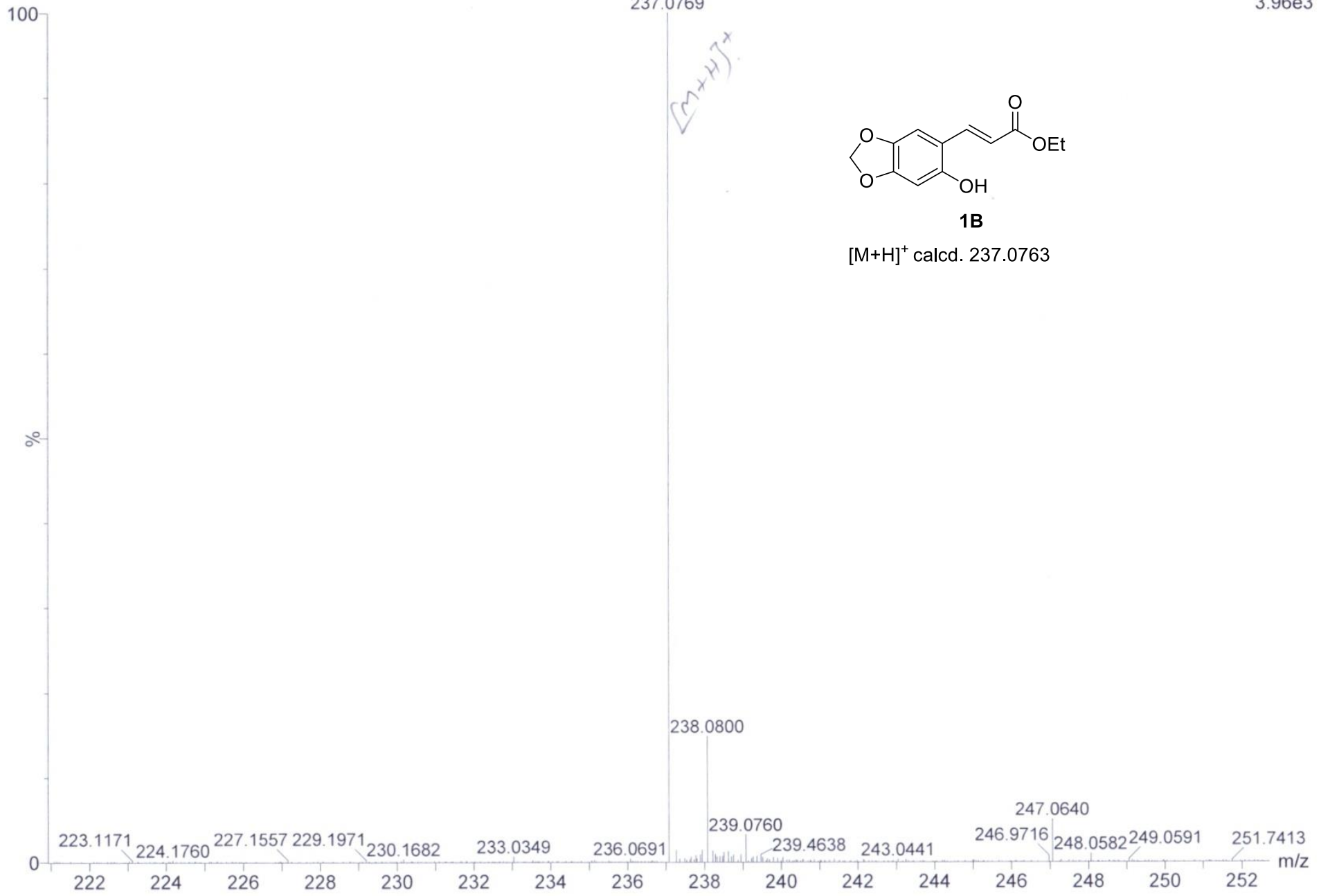

ESI(HRMS) spectrum of (E)-ethyl 3-(6-hydroxybenzo[d][1,3]dioxol-5-yl)acrylate (1B) 
SRK10-136 22 (0.461) AM (Cen,4, 100.00, Ar,8500.0,556.28,1.00,LS 10); Sm (SG, 2x5.00); Sb (10,1.00); Cm (22:23-1:3)

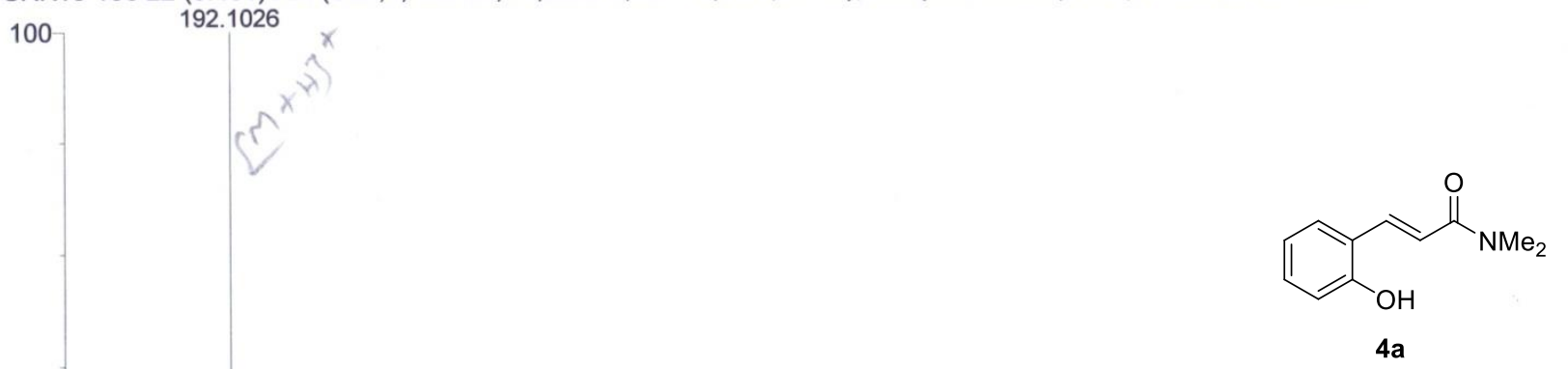

$[\mathrm{M}+\mathrm{H}]^{+}$calcd. 192.1025

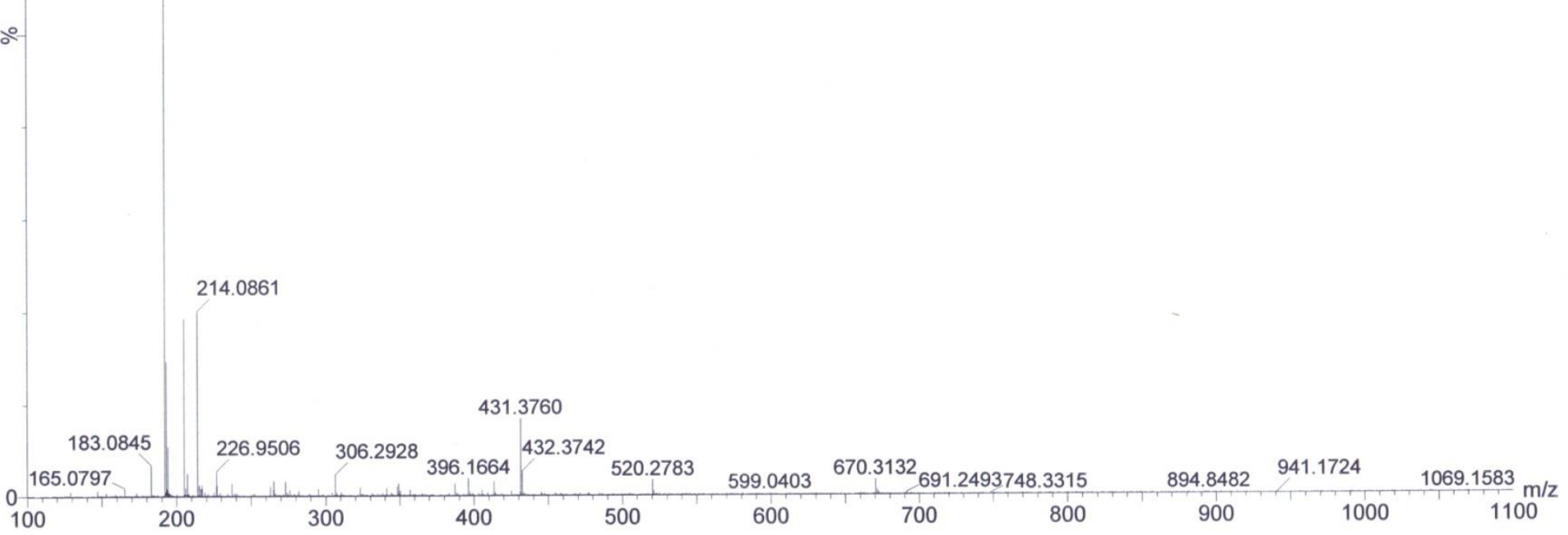

ESI(HRMS) spectrum of (E)-3-(2-hydroxyphenyl)- $N, N$-dimethylacrylamide (4a) 
SRK12-119-II 19 (0.405) AM (Cen,4, 100.00, Ar,8500.0,556.28,1.00,LS 10); Sm (SG, 2x5.00); Sb (10,1.00); Cm (19:20-1:3) 100 210.0935

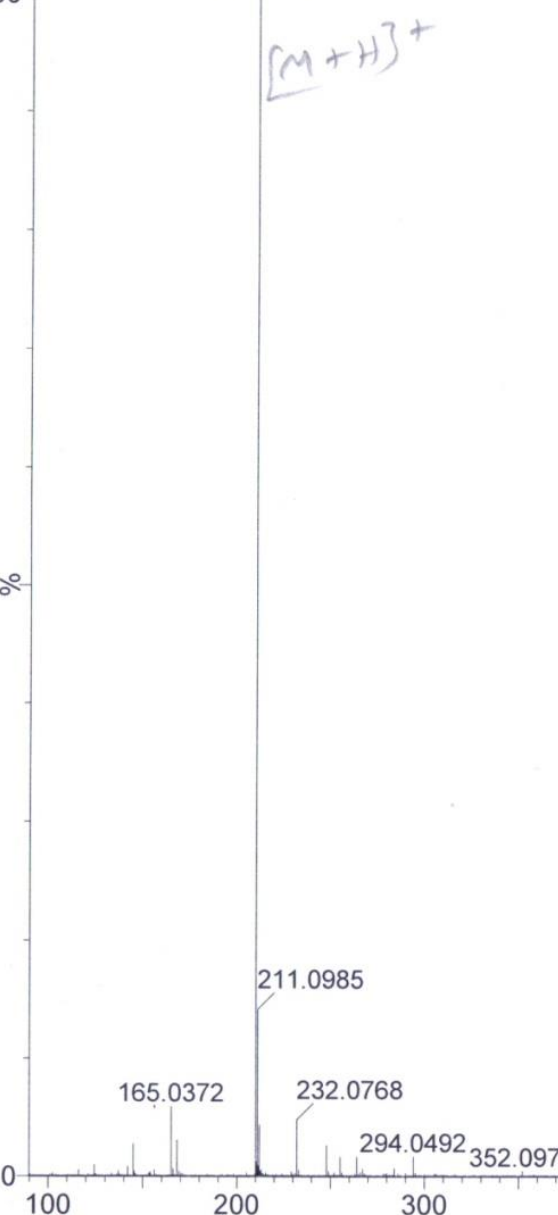

419.1810457 .1247 $509.0905 \quad 650.2448708 .5234729 .1497$

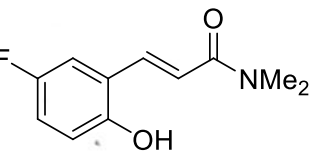

4b

$[\mathrm{M}+\mathrm{H}]^{+}$calcd. 210.0930

ESI(HRMS) spectrum of (E)-3-(5-fluoro-2-hydroxyphenyl)- $N, N$-dimethylacrylamide (4b) 
SRK12-120 19 (0.406) AM (Cen,4, 100.00, Ar,8500.0,556.28,5.00,LS 10); Sm (SG, 2×5.00); Sb (10,1.00); Cm (19:21-1:3) 100 226.0637

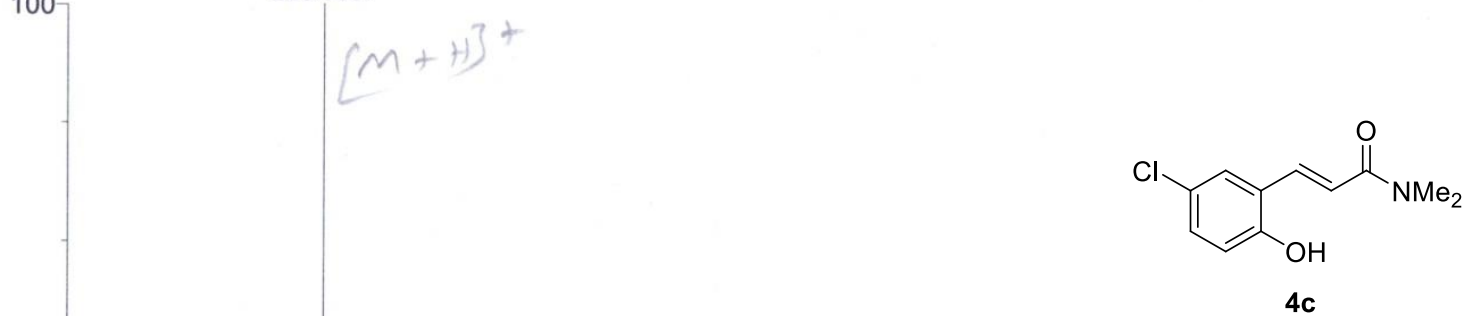

$[\mathrm{M}+\mathrm{H}]^{+}$calcd. 226.0635

228.0623

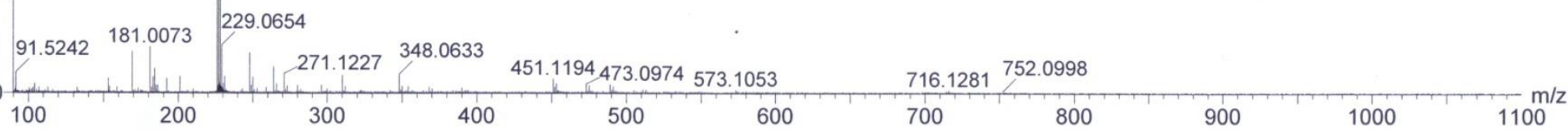

ESI(HRMS) spectrum of (E)-3-(5-chloro-2-hydroxyphenyl)- $N, N$-dimethylacrylamide (4c) 

SRK12-156 19 (0.405) AM (Cen,4, 100.00, Ar,8500.0,556.28,0.75,LS 10); Sm (SG, 2x5.00); Sb (10,1.00); Cm (19:21-1:3)
222.1130

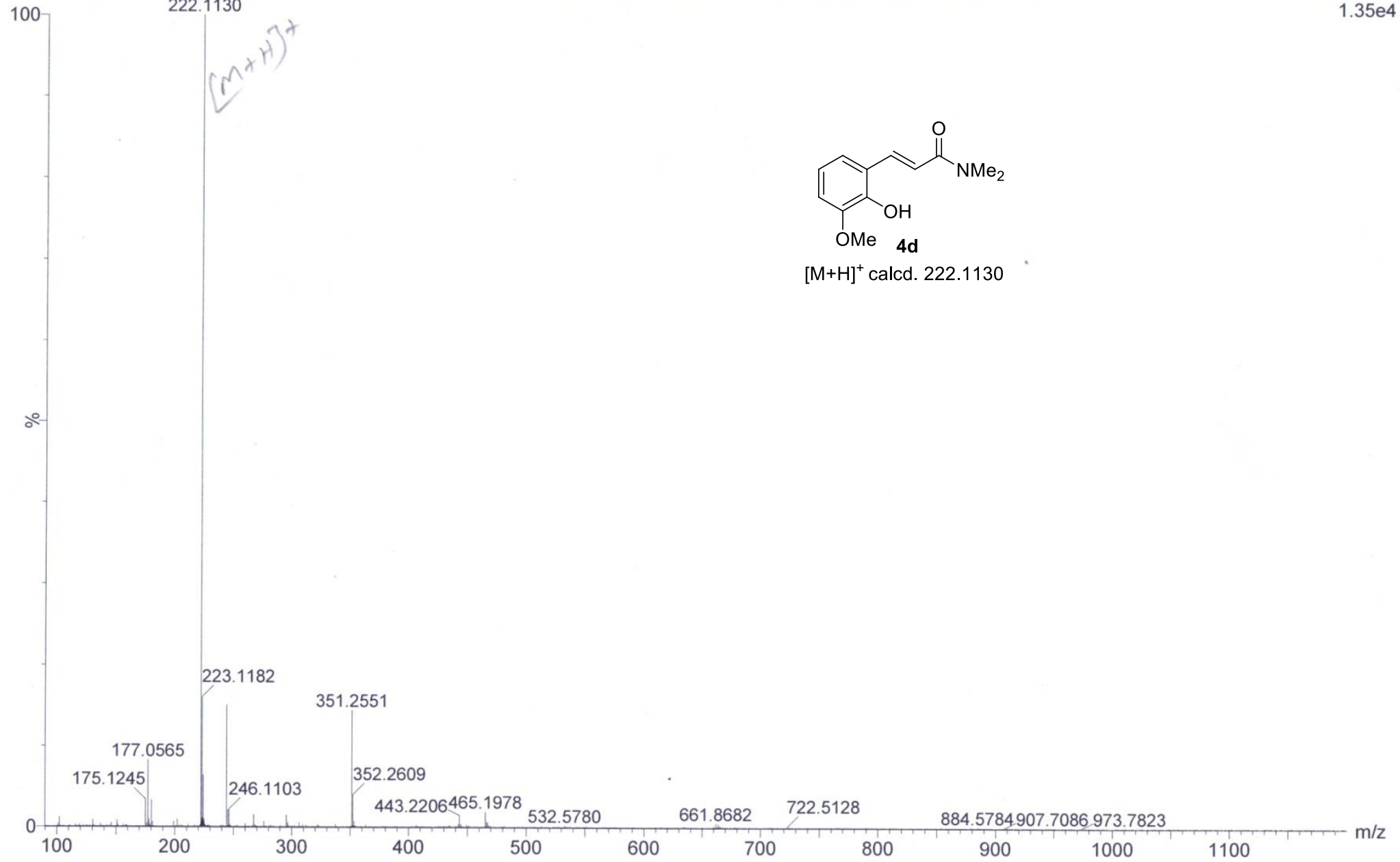

ESI(HRMS) spectrum of (E)-3-(2-hydroxy-3-methoxyphenyl)- $N, N$-dimethylacrylamide (4d) 